\title{
COMMENSURATORS OF CUSPED HYPERBOLIC MANIFOLDS
}

\author{
OLIVER GOODMAN, DAMIAN HEARD, AND CRAIG HODGSON
}

\begin{abstract}
This paper describes a general algorithm for finding the commensurator of a non-arithmetic hyperbolic manifold with cusps, and for deciding when two such manifolds are commensurable. The method is based on some elementary observations regarding horosphere packings and canonical cell decompositions. For example, we use this to find the commensurators of all non-arithmetic hyperbolic once-punctured torus bundles over the circle.

For hyperbolic 3-manifolds, the algorithm has been implemented using Goodman's computer program Snap. We use this to determine the commensurability classes of all cusped hyperbolic 3-manifolds triangulated using at most 7 ideal tetrahedra, and for the complements of hyperbolic knots and links with up to 12 crossings.
\end{abstract}

\section{INTRODUCTION}

Two manifolds or orbifolds $M$ and $M^{\prime}$ are commensurable if they admit a common finite sheeted covering. For hyperbolic $n$-orbifolds, we can suppose that $M=\mathbb{H}^{n} / \Gamma$ and $M^{\prime}=\mathbb{H}^{n} / \Gamma^{\prime}$, with $\Gamma$ and $\Gamma^{\prime}$ discrete subgroups of $\operatorname{Isom}\left(\mathbb{H}^{n}\right)$. In this paper, we assume that $M$ and $M^{\prime}$ are of finite volume and of dimension at least 3. Then, by Mostow-Prasad Rigidity, commensurability means that we can conjugate $\Gamma$ by an isometry $g$ such that $g \Gamma g^{-1}$ and $\Gamma^{\prime}$ intersect in a subgroup of finite index in both groups.

Given that the classification of finite volume hyperbolic manifolds up to homeomorphism appears to be hard, it seems sensible to attempt to subdivide the problem and start with a classification up to commensurability. Looked at in this way, we see a remarkable dichotomy between the arithmetic and non-arithmetic cases. (See 23 for the definition of arithmetic hyperbolic manifolds.)

Define the commensurator of $\Gamma$ to be the group

$$
\operatorname{Comm}(\Gamma)=\left\{g \in \operatorname{Isom}\left(\mathbb{H}^{n}\right) \mid\left[\Gamma: \Gamma \cap g \Gamma g^{-1}\right]<\infty\right\} .
$$

Then $\Gamma$ and $\Gamma^{\prime}$ are commensurable if and only if $\operatorname{Comm}(\Gamma)$ and $\operatorname{Comm}\left(\Gamma^{\prime}\right)$ are conjugate. Geometrically, an element of the normalizer of $\Gamma$ in $\operatorname{Isom}\left(\mathbb{H}^{n}\right)$ represents a symmetry (i.e. isometry) of $M=\mathbb{H}^{n} / \Gamma$. Similarly, an element of the commensurator represents an isometry between finite sheeted covers of $M$; this gives a hidden symmetry of $M$ if it is not the lift of an isometry of $M$ (see [27]).

It follows from deep work of Margulis [24] (see also [35]), that in dimension $\geq 3$, the commensurator $\operatorname{Comm}(\Gamma)$ is discrete if and only if $\Gamma$ is not arithmetic. This means that the commensurability class of a non-arithmetic, cofinite volume, discrete group $\Gamma$ is particularly simple, consisting only of conjugates of the finite index subgroups of $\operatorname{Comm}(\Gamma)$. In terms of orbifolds, it means that $M$ and $M^{\prime}$ are commensurable if and only if they cover a common quotient orbifold.

On the other hand, commensurability classes of arithmetic groups are "big": we may well have commensurable $\Gamma$ and $\Gamma^{\prime}$ such that the group generated by $g \Gamma g^{-1}$ and $\Gamma^{\prime}$ is not discrete for any $g$.

This work was partially supported by grants from the Australian Research Council. 
A hyperbolic $n$-orbifold is cusped if it is non-compact of finite volume. This paper describes a practical algorithm for determining when two cusped hyperbolic $n$-manifolds cover a common quotient, and for finding a smallest quotient. For non-arithmetic finite volume cusped hyperbolic $n$-manifolds of dimension $n \geq 3$, this solves the commensurability problem.

Section 2 begins with some elementary observations about horoball packings and canonical cell decompositions of a cusped hyperbolic manifold. This leads to a characterization of the commensurator of a non-arithmetic cusped hyperbolic manifold $M$ as the maximal symmetry group of the tilings of $\mathbb{H}^{n}$ obtained by lifting canonical cell decompositions of $M$. In Section 3, we use this to determine the commensurators of non-arithmetic hyperbolic once-punctured torus bundles over the circle.

Section 4 gives an algorithm for finding the isometry group of a tiling of $\mathbb{H}^{n}$ arising from a cell decomposition of a hyperbolic manifold, and Sections 5 and 6 describe methods for finding all possible canonical cell decompositions for a cusped hyperbolic manifold. Section 7 contains some observations on commensurability of cusps in hyperbolic 3-manifolds which can simplify the search for all canonical cell decompositions.

In 3-dimensions, each orientable hyperbolic orbifold has the form $M=\mathbb{H}^{3} / \Gamma$, where $\Gamma$ is a discrete subgroup of $\operatorname{PSL}(2, \mathbb{C})=\operatorname{Isom}^{+}\left(\mathbb{H}^{3}\right)$. The invariant trace field $k(\Gamma) \subset \mathbb{C}$ is the field generated by the traces of the elements of $\Gamma^{(2)}=\left\{\gamma^{2} \mid \gamma \in \Gamma\right\}$ lifted to $\operatorname{SL}(2, \mathbb{C})$. This is a number field if $M$ has finite volume (see [27, [29], $[22]$ ). The invariant quaternion algebra is the $k(\Gamma)$ subalgebra of $M_{2}(\mathbb{C})$ generated by $\Gamma^{(2)}$. These are useful and computable commensurability invariants (see [10], [23]).

For the arithmetic subgroups of $\operatorname{Isom}\left(\mathbb{H}^{3}\right)$, the invariant quaternion algebra is a complete commensurability invariant. In fact for cusped arithmetic hyperbolic 3orbifolds, the invariant trace field is an imaginary quadratic field and the quaternion algebra is just the algebra of all $2 \times 2$ matrices with entries in the invariant trace field (see [23, Theorem 3.3.8]); so the invariant trace field is a complete commensurability invariant. However most cusped hyperbolic 3-manifolds are non-arithmetic (cf. [6]) so other methods are needed to determine commensurability.

Damian Heard and Oliver Goodman have implemented the algorithms described in this paper for non-arithmetic hyperbolic 3-manifolds; these are incorporated in the computer program Snap [16. Using this we have determined the commensurability classes for all manifolds occurring in the Callahan-Hildebrand-Weeks census ([19], [9]) of cusped hyperbolic manifolds with up to 7 tetrahedra, and for complements of hyperbolic knots and links up to 12 crossings, supplied by Morwen Thistlethwaite (see 20]). These results are discussed in Section 8, while Section 9 outlines the Dowker-Thistlethwaite notation used to describe links.

This work has uncovered interesting new examples of commensurable knot and link complements (see Examples 2.1 and 2.2), and a new example of a knot with shape field properly contained in the invariant trace field (see Example 7.1). The results have also been used by Button 8 , to study fibred and virtually fibred cusped hyperbolic 3-manifolds.

For 1-cusped manifolds we note that "cusp density" (see Section 22 is a very good invariant. We have found only a few examples of incommensurable 1-cusped manifolds which are not distinguished by cusp density (see Example 2.3).

There is also a "dumb" algorithm, based on volume bounds for hyperbolic orbifolds, which works for any (possibly closed) non-arithmetic hyperbolic 3-orbifold, but appears to be quite impractical. If $M$ and $M^{\prime}$ cover $Q$ with $\operatorname{Vol}(Q)>C$ then the degrees $d, d^{\prime}$ of the coverings are bounded by $D=\lfloor\operatorname{Vol}(M) / C\rfloor$ and 
$D^{\prime}=\left\lfloor\operatorname{Vol}\left(M^{\prime}\right) / C\right\rfloor$, respectively. Then if $M$ and $M^{\prime}$ are commensurable, they admit a common covering $N$ of degree at most $D^{\prime}$ over $M$ and at most $D$ over $M^{\prime}$. The best current estimate for $C$ for orientable non-arithmetic 3-orbifolds is $0.041 \ldots$ from recent work of Marshall-Martin [25. Since $\operatorname{Vol}(M) \approx 2$ is typical, we would have to find all coverings of $M^{\prime}$ of degree $d^{\prime} \leq 50$. This means finding all conjugacy classes of transitive representations of $\pi_{1}\left(M^{\prime}\right)$ into $S_{50}$, a group with around $10^{64}$ elements!

Acknowledgements: We thank Ian Agol for pointing out a simplification to our method of determining the commensurability of Euclidean tori, Gaven Martin for information on current volume bounds, and Walter Neumann for several interesting discussions on this work. We also thank Alan Reid, Genevieve Walsh, and the referee for their helpful comments on the paper.

\section{The Commensurability Criterion}

We use the following terminology throughout this paper. A set of disjoint horoballs in $\mathbb{H}^{n}$ is called a horoball packing, and a cusp neighbourhood in a hyperbolic $n$-orbifold is one which lifts to such a horoball packing.

Lemma 2.1. The symmetry group of a horoball packing in $\mathbb{H}^{n}$ is discrete whenever the totally geodesic subspace spanned by their ideal points has dimension at least $n-1$.

Proof. Let $\left\{g_{i}\right\}$ be a sequence of symmetries of the packing converging to the identity. Choose horoballs $B_{1}, \ldots, B_{n}$ whose ideal points span a totally geodesic subspace $H$ of dimension $n-1$. For $i$ sufficiently large, we can assume that $g_{i}\left(B_{k}\right)=B_{k}$ for $k=1, \ldots, n$. But this implies that these $g_{i}$ fix $H$ pointwise. Since the only such isometries are the identity, and reflection in $H$, the sequence must be eventually constant.

Lemma 2.2. Let $M=\mathbb{H}^{n} / \Gamma$ be a finite volume cusped hyperbolic orbifold. The set of parabolic fixed points of $\Gamma$ spans $\mathbb{H}^{n}$.

Proof. The set of parabolic fixed points is dense in the limit set of $\Gamma$ which equals the whole of the sphere at infinity.

Lemma 2.3. Let $M, M^{\prime}$ be finite volume cusped hyperbolic orbifolds. Then $M$ and $M^{\prime}$ cover a common orbifold $Q$ if and only if they admit choices of cusp neighbourhoods lifting to isometric horoball packings.

Proof. If $M$ and $M^{\prime}$ cover $Q$, choose cusp neighbourhoods in $Q$ and lift to $M$ and $M^{\prime}$. These all lift to the same horoball packing in $\mathbb{H}^{n}$, namely the horoball packing determined by our choice of cusp neighbourhoods in $Q$. Conversely, both $M$ and $M^{\prime}$ cover the quotient of $\mathbb{H}^{n}$ by the group of symmetries of their common horoball packing which, by Lemmas 2.1 and 2.2, is discrete.

We can define the cusp density of a 1-cusped hyperbolic orbifold $M$ as follows. Since $M$ has only one cusp it has a unique maximal (embedded) cusp neighbourhood $U$. The cusp density of $M$ is $\operatorname{Vol}(U) / \operatorname{Vol}(M)$. Since the cusp density of any orbifold covered by $M$ is the same, it is a commensurability invariant of orbifolds with discrete commensurator.

Choosing a full set of disjoint cusp neighbourhoods in a non-compact finite volume hyperbolic $n$-manifold $M$ determines a "Ford spine." This is the cell complex given by the set of points in $M$ equidistant from the cusp neighbourhoods in two or more directions. Cells of dimension $n-k$ contain points equidistant from the cusp neighbourhoods in $k+1$ independent directions $(k=1, \ldots, n)$. This spine can 
also be seen intuitively as the "bumping locus" of the cusp neighbourhoods: blow up the cusp neighbourhoods until they press against each other and flatten.

Dual to the Ford spine is a decomposition of $M$ into ideal polytopes, generically simplices. The ideal cell dual to a given 0-cell of the Ford spine lifts to the convex hull in $\mathbb{H}^{n}$ of the set of ideal points determined by the equidistant directions. We call the cell decompositions that arise in this way canonical 1 For a 1-cusped manifold the canonical cell decomposition is unique. It is shown in [4] that a finite volume hyperbolic manifold with multiple cusps admits finitely many canonical cell decompositions.

Theorem 2.4. Cusped hyperbolic n-manifolds $M$ and $M^{\prime}$ cover a common orbifold if and only if they admit canonical ideal cell decompositions lifting to isometric tilings of $\mathbb{H}^{n}$.

Proof. If $M$ and $M^{\prime}$ cover $Q$, choose cusp neighbourhoods in $Q$ and lift them to $M, M^{\prime}$ and $\mathbb{H}^{n}$. Constructing the Ford spine and cell decomposition in $\mathbb{H}^{n}$ clearly yields the lifts of those entities from both $M$ and $M^{\prime}$ corresponding to our choice of cusp neighbourhoods.

Conversely, observe that the symmetry group of the common tiling gives an orbifold which is a quotient of both manifolds.

Remark 2.5. We can omit the word 'canonical' in the above theorem. The proof is unchanged.

The previous theorem gives the following characterization of the commensurator.

Theorem 2.6. Let $M=\mathbb{H}^{n} / \Gamma$ be a finite volume cusped hyperbolic $n$-manifold with discrete commensurator. Then $\operatorname{Comm}(\Gamma)$ is the maximal symmetry group of the tilings of $\mathbb{H}^{n}$ obtained by lifting canonical cell decompositions of $M$; it contains all such symmetry groups.

For manifolds with discrete commensurator, we can now define a truly canonical ideal cell decomposition as follows. Find $\operatorname{Comm}(\Gamma)$ as in the above theorem. Choose equal volume cusp neighbourhoods in $\mathbb{H}^{n} / \operatorname{Comm}(\Gamma)$. Lift them to $M$ and take the resulting canonical cell decomposition of $M$. Two such manifolds are commensurable if and only if their truly canonical cell decompositions give isometric tilings of $\mathbb{H}^{n}$.

Choosing maximal cusp neighbourhoods in $\mathbb{H}^{n} / \operatorname{Comm}(\Gamma)$ also gives a canonical version of cusp density for multi-cusped manifolds.

Theorem 2.6 is the basis for the algorithms described in this paper. Canonical cell decompositions can be computed by the algorithms of Weeks described in 33. and implemented in SnapPea [34. In Section 4 below we give an algorithm for finding the isometry groups of the corresponding tilings of $\mathbb{H}^{n}$. Combining this with Theorem 2.6 gives an algorithm for finding commensurators of 1-cusped non-arithmetic hyperbolic $n$-manifolds. In Sections 5 and 6 we extend this algorithm to multi-cusped manifolds, by describing methods for finding all canonical cell decompositions.

For hyperbolic 3-manifolds, these algorithms have been implemented by Heard and Goodman. (These are incorporated in "find commensurator" and related commands in the program Snap [16]). We conclude this section with some examples discovered during this work.

\footnotetext{
${ }^{1}$ The term is not really ideal since, for manifolds with multiple cusps, there are generally multiple canonical cell decompositions depending on the choice of cusp neighbourhoods.
} 
2.1. Example: a 5-link chain and friends. The following five links have commensurable complements, as shown using our computer program. In the first three cases at least it is possible to 'see' this commensurability.
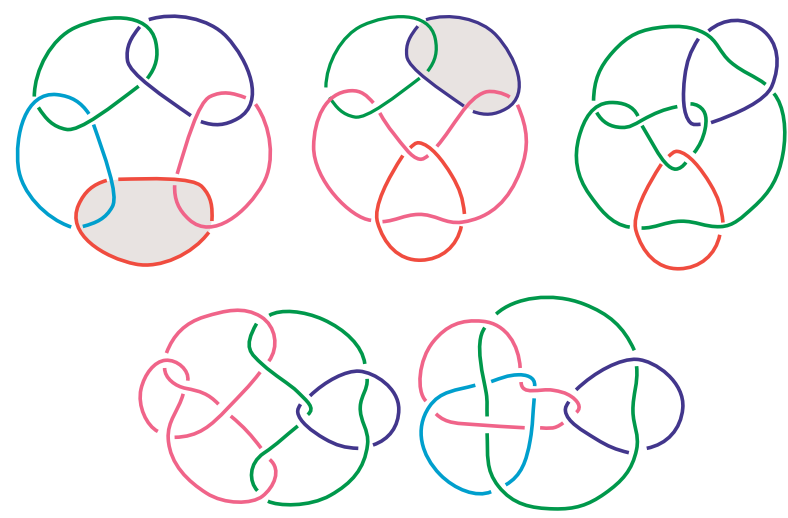

The first of these is the 5 -link chain $C_{5}$. Thurston [32, Chapter 6 ] explains how to obtain a fundamental region for the hyperbolic $k$-link chain complements: we span each link of the chain by a disk in the obvious manner. The complement of the union of these five disks is then a solid torus. Once the link is deleted, the disks and their arcs of intersection divide the boundary of the solid torus into ideal squares $A, B, C, D, E$ as shown, with cusps labelled $a, b, c, d, e$.

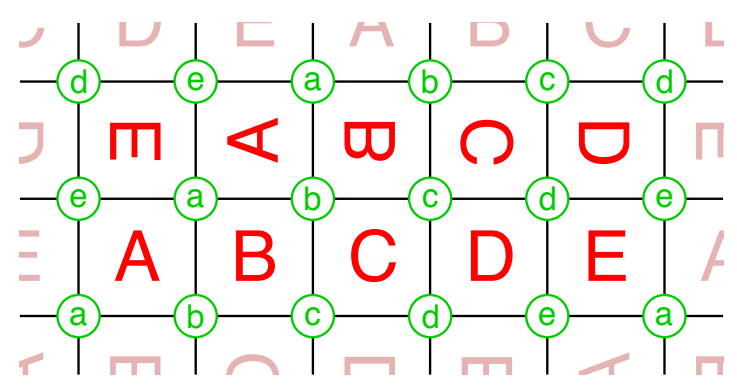

A hyperbolic structure is given by taking two regular pentagonal drums with ideal vertices and adjusting their heights to obtain (ideal) square faces. Glue two drums together as shown, identify the top with the bottom via a $\frac{4 \pi}{5}$ rotation, and glue faces as indicated. Edges are then identified in 4's, two horizontal with two vertical. It is easy to check that the sum of dihedral angles around each edge is $2 \pi$ so this gives a hyperbolic structure, since the angle sum is $\pi$ at each ideal vertex of a drum.

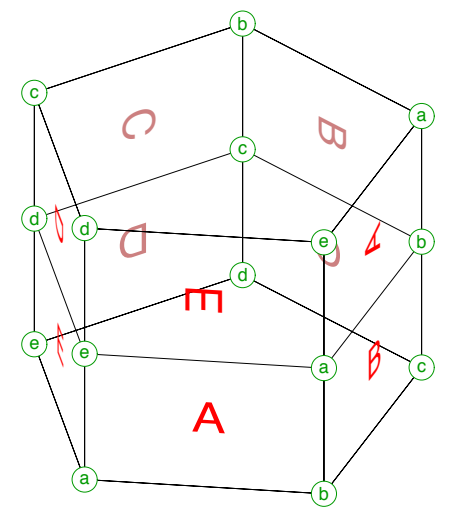


It is clear from the symmetry of the picture that the drums are cells in the canonical cell decomposition obtained by choosing equal area cusp cross sections. We remark that Neumann-Reid show that this link complement is non-arithmetic in [27. Section 5].

Now change the 5-link chain by cutting along the shown disk, applying a half twist, and re-gluing to obtain our second link. In the complement, this surgery introduces a half turn into the gluing between the $A$-faces. Edges are still identified in 4's, two horizontal with two vertical, but there are now only four cusps.

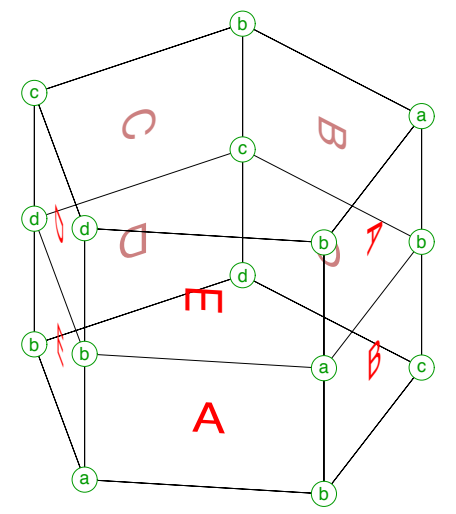

If we repeat the process on the second disk shown we obtain the third link. Again this corresponds to changing the gluing pattern on our two drums.

Since these link complements are non-arithmetic, the tiling of $\mathbb{H}^{3}$ by pentagonal drums covers some canonical cell decomposition of each one. Since their volumes are the same, each one decomposes into two pentagonal drums. It should therefore be possible, in each case, to find 5 ideal squares meeting at order 4 edges, cutting the complement into one or two solid tori. We leave this as a challenge for the reader.

2.2. Example: commensurable knot complements. Commensurable knot complements seem to be rather rare. Previously known examples include the RubinsteinAitchison dodecahedral knots [3] and examples due to a construction of GonzálesAcuña and Whitten 13 giving knot complements covering other knot complements. For example, the $-2,3,7$ pretzel knot has 18/1 and 19/1 surgeries giving lens spaces. Taking the universal covers of these lens spaces gives new hyperbolic knots in $S^{3}$ whose complements are 18- and 19-fold cylic covers of the $(-2,3,7)$-pretzel complement.

Our program finds a pair of knots " $9 \mathrm{n} 6$ " and " $12 \mathrm{n} 642$ ", having 9 and 12 crossings respectively, whose complements are commensurable with volumes in the ratio $3: 4$. Walter Neumann has pointed out that these knots belong to a very pretty family of knots: take a band of $k$ repeats of a trefoil with the ends given $m$ half twists before putting them together. E.g. $(k, m)=(3,2)$ :

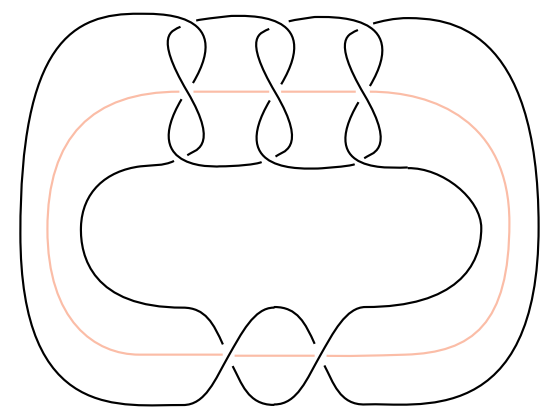


The half-twists are put in so as to undo some of the crossings of the trefoils (allowing the above projection to be rearranged so as to have 9 crossings). The pair of knots found by our program correspond to $(k, m)=(3,2)$ and $(4,1)$.

To see that these knots have commensurable complements we find a common quotient orbifold. In each case this is the quotient of the knot complement by its symmetry group; these are dihedral groups of order 12 and order 16 respectively.

The picture of $9 n 6$ above shows an obvious axis of 2-fold symmetry; below left is the quotient, which is the complement of a knot in the orbifold $S^{3}$ with singular set an unknot labelled 2. By pulling the knot straight, we see that this is an orbifold whose underlying space is a solid torus with knotted singular locus.
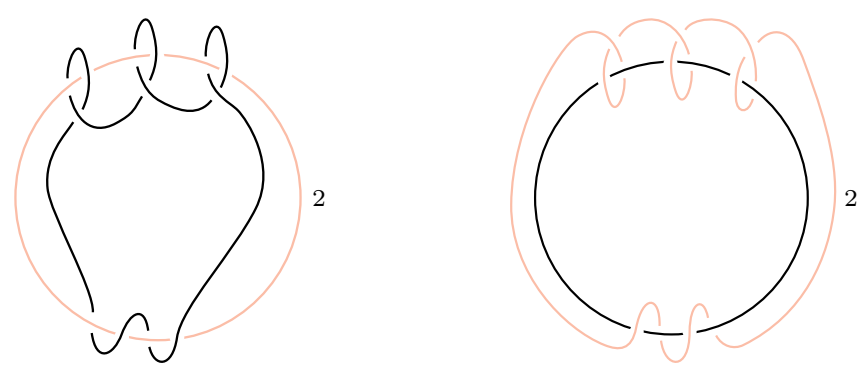

If we arrange the singular locus on a torus parallel to the boundary of the solid torus we see 3 clasps, 3 strands in the (vertical) core direction, and a strand with slope $2 / 1$. The view from inside the solid torus looking towards the boundary is shown below. (For the knot $(4,1)$ we would see 4 clasps, 4 strands in the core direction and a strand with slope $1 / 1$.)

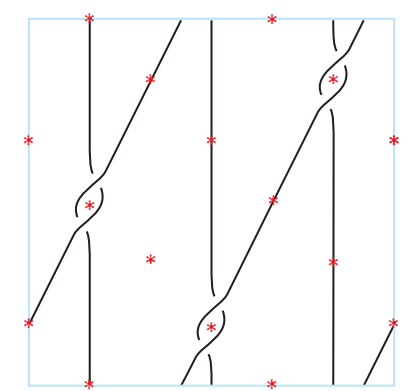

The solid torus with its singular set has three 2-fold symmetries whose axes intersect the solid torus in 6 arcs, each passing perpendicularly through the core like a skewer, with symmetry group dihedral of order 6 . The ends of the arcs are shown as stars above.

The quotient orbifold is obtained by taking a slice of the solid torus between two axes and folding closed the top and bottom disks like books. The result is a ball with the axes giving two unknotted arcs of order 2 in the singular set, running out to the boundary (which is now a $(2,2,2,2)$-pillowcase orbifold). The original singular set gives an arc linking the other two, so that the whole singular locus is an 'H' graph labelled with 2's.

The last three pictures show what happens to the singular locus in one slice of the solid torus as we fold. We begin with the annulus in the bottom $1 / 6$ th of the previous figure, redrawn after twisting the bottom. This bounds a solid cylinder with the singular locus as shown in the middle figure. Folding along the top and 
bottom (and expanding the region slightly) gives the final result.
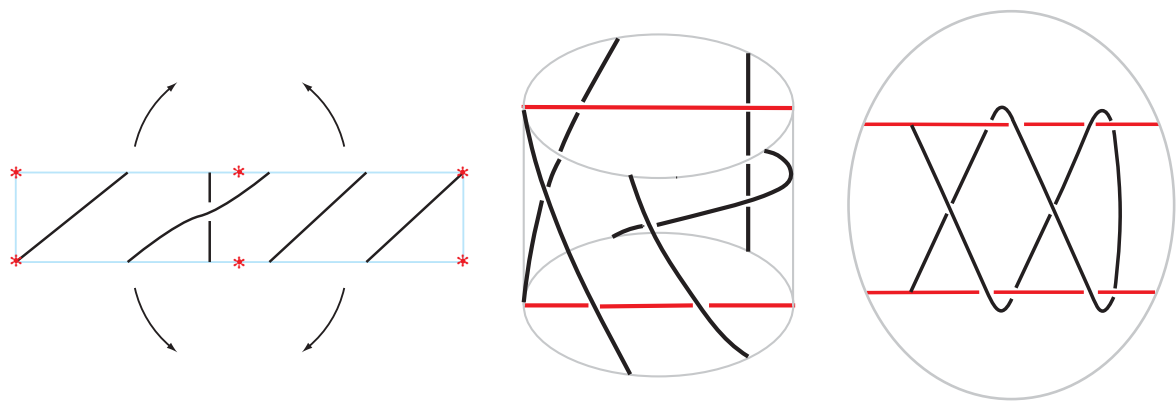

We leave it to the reader to draw similar pictures for the knot with $(k, m)=(4,1)$ and verify that the result is indeed the same orbifold. Alternatively, Orb [18 or SnapPea 34 can be used to verify that the appropriate dihedral covers of the final orbifold give the complements of the knots with $(k, m)=(3,2)$ and $(4,1)$.

We remark that Walter Neumann has found an infinite family of new examples of pairs of commensurable knot complements in the 3 -sphere; this example is the simplest case.

2.3. Example: cusp horoball pictures. Figure 1 shows the horoball packings of two 1-cusped census manifolds m137 and m138 as seen from the cusp. Using Snap [16], we find that the commensurability classes of these two equal-volume manifolds are indistinguishable by cusp density or invariant trace field. Their maximal horoball packings and canonical cell decompositions are however different. (For example, the edges joining degree 4 vertices in the following cusp diagrams are all parallel for $\mathrm{m} 137$, but not for $\mathrm{m} 138$.)

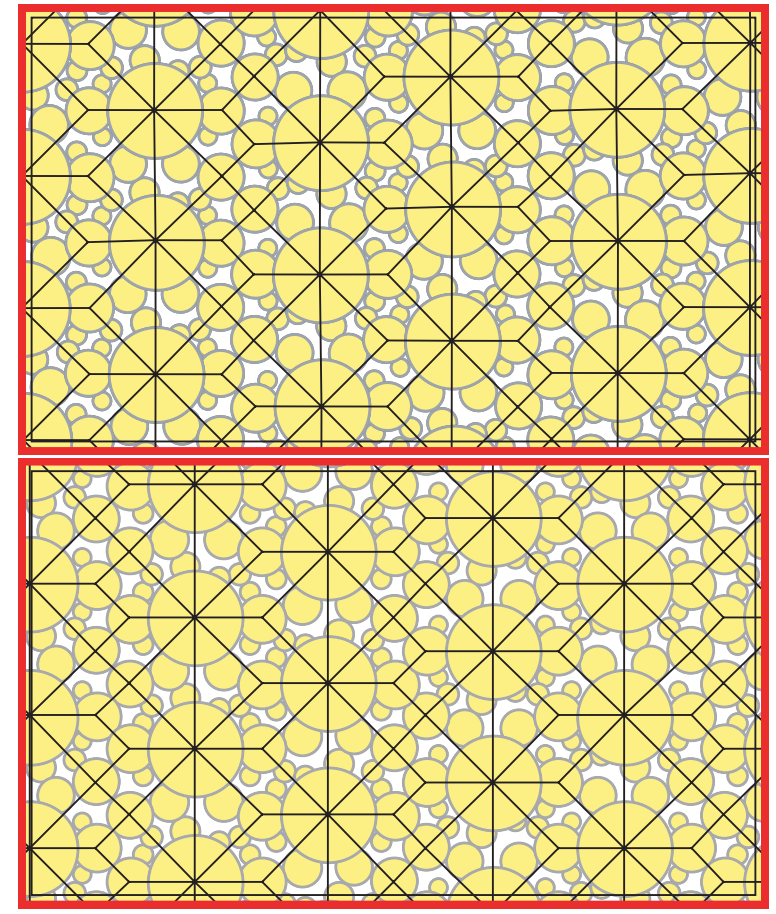

FiguRE 1. The maximal horoball packing and canonical cell decomposition as seen from the cusps of manifolds m137 and m138. 


\section{EXAMPle: PUNCTURED TORUS BUNDLES}

The bundles over $S^{1}$ with a once-punctured torus as fibre provide an interesting family of 1-cusped hyperbolic 3-manifolds with known canonical triangulations. By analysing how symmetries of the lifted triangulations of the universal cover appear when viewed from the cusp we obtain (in all the non-arithmetic cases) strong constraints on what symmetries may be possible. We then show that they all come from symmetries of the manifold. This leads to the following:

Theorem 3.1. Let $M=\mathbb{H}^{3} / G$ be an orientable non-arithmetic hyperbolic 3manifold which is a once-punctured torus bundle over $S^{1}$. Then $M$ has no "hidden symmetries", i.e. the commensurator of $G$ is the normalizer of $G$ in $\operatorname{Isom}\left(\mathbb{H}^{3}\right)$.

Let $F$ denote a once-punctured torus, and let $\varphi: F \rightarrow F$ be an orientation preserving homeomorphism. Let

$$
M=M_{\varphi}=F \times_{\varphi} S^{1}=\frac{F \times[0,1]}{(x, 0) \sim(\varphi(x), 1)}
$$

be the mapping torus of $\varphi$. Identifying $F$ with $\left(\mathbb{R}^{2}-\mathbb{Z}^{2}\right) / \mathbb{Z}^{2}$, we have that $\varphi$ is isotopic to an element of $\operatorname{SL}(2, \mathbb{Z})$; since $M$ depends only on the isotopy class of $\varphi$ we assume $\varphi \in \operatorname{SL}(2, \mathbb{Z})$. Then $M$ is hyperbolic whenever $\varphi$ is hyperbolic, i.e. when $\varphi$ has distinct real eigenvalues; $M_{\varphi}$ and $M_{\varphi^{\prime}}$ are homeomorphic if and only if $\varphi$ and $\varphi^{\prime}$ are conjugate.

Define matrices

$$
L=\left(\begin{array}{ll}
1 & 0 \\
1 & 1
\end{array}\right), \quad R=\left(\begin{array}{ll}
1 & 1 \\
0 & 1
\end{array}\right) .
$$

For each word $w$ in the symbols $L, R$ define $\varphi_{w} \in \mathrm{SL}(2, \mathbb{Z})$ as the corresponding matrix product.

Lemma 3.2. Each $\varphi \in S L(2, \mathbb{Z})$ is conjugate to $\pm \varphi_{w}$ for some word $w$ in the symbols $L, R$. The sign is unique and $w$ is determined up to cyclic permutations of its letters.

Let $M=M_{\varphi}$ where $\varphi= \pm \varphi_{w}$ is hyperbolic. The so-called monodromy triangulation $T$ of $M$ has one tetrahedron for each letter in $w$ and gluings determined by $w$ and the sign. It is nicely described in [12] and 14. It follows from work of Lackenby [21] that $T$ is the canonical ideal cell decomposition of $M$. Other proofs of this result have recently been given by Guéritaud ([14], 15]) and Akiyoshi, Sakuma, Wada and Yamashita (see [5]).

The intersection of $T$ with a (small) torus cross section of the cusp of $M$, lifted to its universal cover $\mathbb{R}^{2}$, gives the (lifted) cusp triangulation $T_{0}$ of $M$. Note that edges and vertices of $T_{0}$ correspond to edges of $T$ seen transversely or end-on respectively. SnapPea 34 provides pictures of these cusp triangulations: see Figure 3 for an example.

We need two things: the first is a combinatorial description of $T_{0}$ in terms of $w$; the second is an understanding of which edges and vertices of $T_{0}$ correspond to the same edges of $T$ in $M$. Both are outlined briefly here: for detailed explanations we refer the reader to the Appendix of [12] and Sections 3 and 4 of [14].

3.1. The monodromy triangulation $T$. The triangulation $T$ is built up in layers by gluing tetrahedra according to the letters of $w$. We begin with an almost flat ideal tetrahedron projecting onto a punctured torus; the tetrahedron has edges $a, b, c, c_{-}$identified as shown below. 


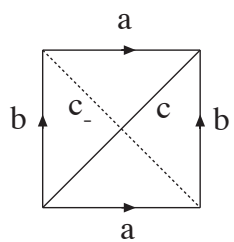

For each successive letter $L$ or $R$ in the word $w$, we attach a tetrahedron to the top of the previous tetrahedron, as shown below in the cover $\left(\mathbb{R}^{2}-\mathbb{Z}^{2}\right) \times \mathbb{R}$.
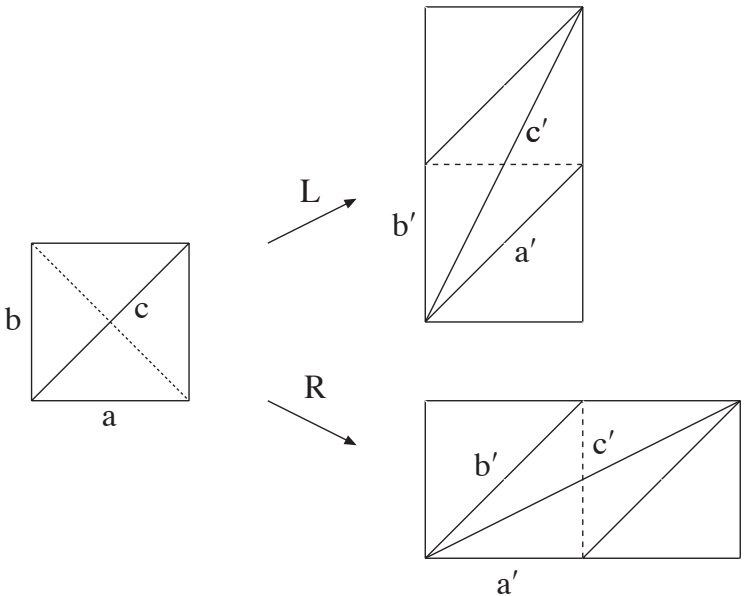

After using all the letters of $w$, the final triangulation of the fibre $F$ differs from the initial triangulation by the monodromy $\varphi$, and we can glue the top and bottom together to obtain an ideal triangulation $T$ of $M$.

3.2. Combinatorial description of $T_{0}$. Now consider the induced triangulation of a cusp linking torus (i.e. cusp cross section) in $M$. Each tetrahedron contributes a chain of 4 triangles going once around the cusp as shown in Figure 2 .
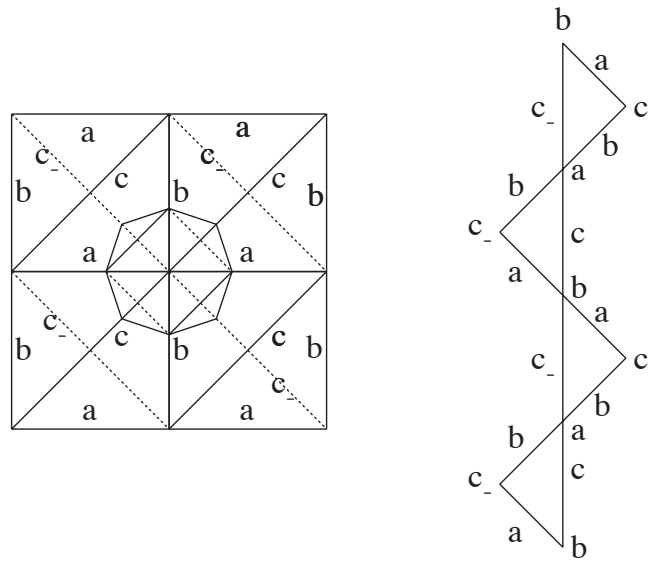

FIGURE 2. The chain of triangles around a cusp coming from one tetrahedron.

In the triangulation $T_{0}$ of $\mathbb{R}^{2}$ this lifts to an infinite chain of triangles forming a (vertical) saw-tooth pattern. 
Each chain is glued to the next in one of two ways depending on whether the letter is an $L$ or an $R$.
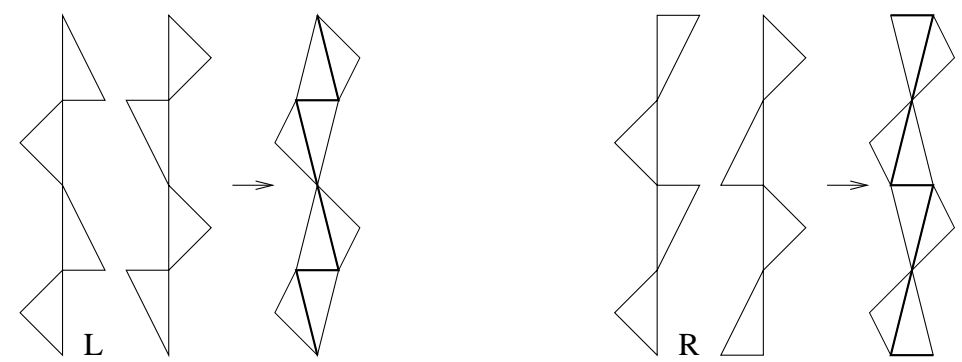

Before gluing we adjust slightly the "front" triangles of the first chain and the "back" triangles of the second, so as to create two horizontal edges. After stacking these chains of triangles together we have a decomposition of $T_{0}$ into horizontal strips which can be described combinatorially as follows.

Make a horizontal strip out of triangles corresponding to the letters of $w$ : for each $L$ add a triangle with a side on the bottom edge of the strip and a vertex at the top; vice-versa for $R$; repeat infinitely in both directions. For example, for $L R R L R$ we have:

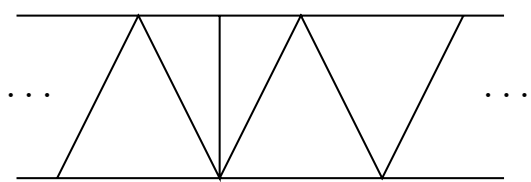

Fill the plane by reflecting repeatedly in the top and bottom edges of the strip. Then the cusp linking torus is the quotient of the plane by the group $G_{0}$ generated by vertical translation by four strips, and horizontal translation by one period of the strip (composed with an extra vertical translation by two strips if $\varphi=-\varphi_{w}$ ).

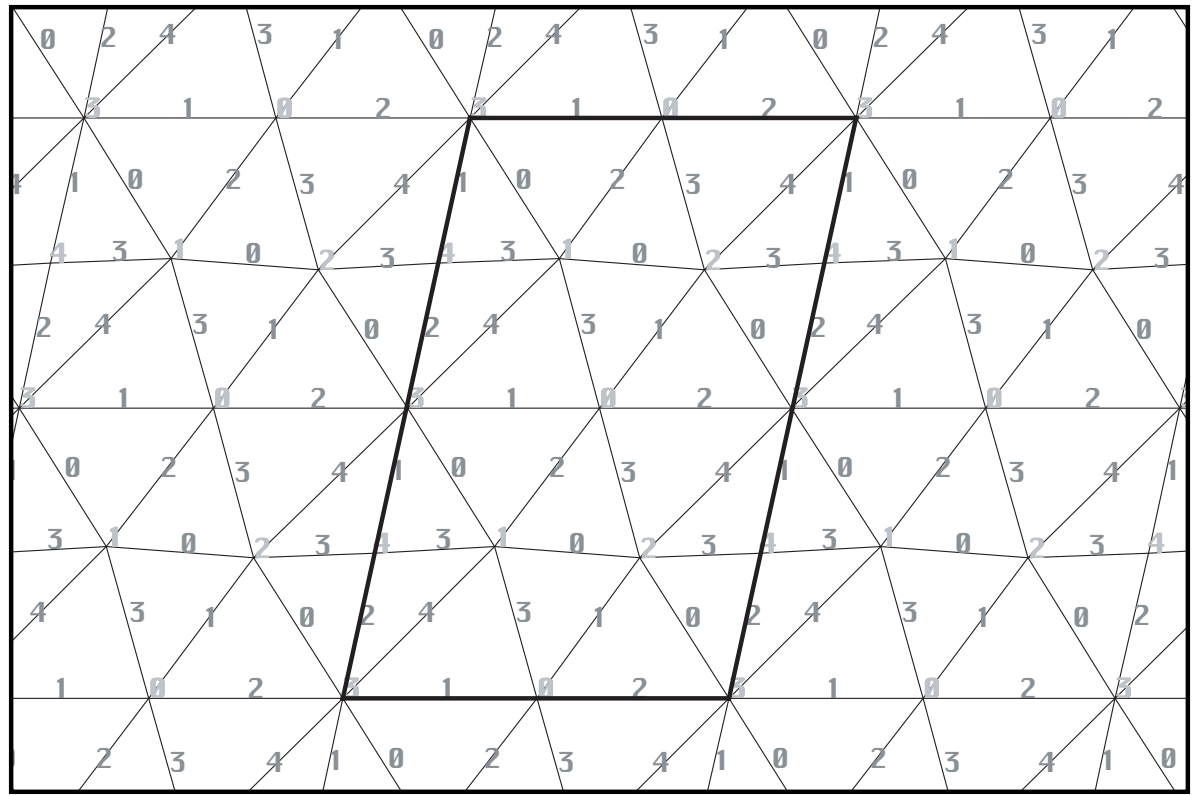

FiguRE 3. The cusp triangulation of $M=M_{\varphi_{w}}$ where $w=$ $L R R L R$. The triangulation of $M$ has five edges, labelled 0-4, which appear as both edges and vertices of the cusp triangulation. 
3.3. Edge/vertex correspondence in $T_{0}$. To understand which edges of $T_{0}$ are identified in $T$, we define a "direction" on each horizontal strip: right to left on the first strip, left to right on the strips below and above it, and so on, so that adjacent strips have opposite directions. Then we have:

Lemma 3.3. Each edge e of $T_{0}$ which crosses a strip bounds two triangles, one of which, $\delta$ say, lies on the side of e given by the direction on the strip. Then $e$ and the opposite vertex of $\delta$ give the same edge of $T$. Horizontal edges of $T_{0}$ correspond with the edges at the opposite vertices of both adjacent triangles.

This result is illustrated in Figure 4 and will be important in the arguments below.

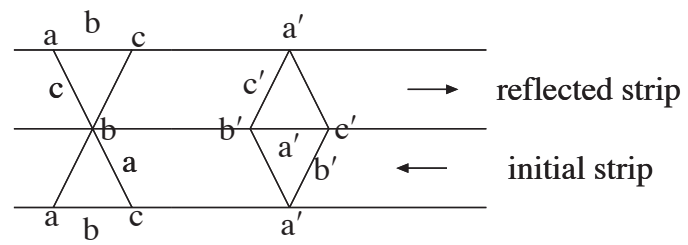

Figure 4. Edge identifications in strips of $T_{0}$.

Proof. The boundary between two tetrahedra in $T$ is a punctured torus consisting of two ideal triangles, homotopic to a fibre of $M$. In $T_{0}$ the boundaries give edge cycles, lifts of a cycle of 6 edges going once around the cusp in the vertical direction as in Figure 2, Here are two cycles:

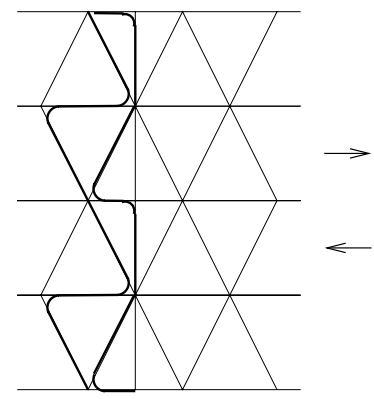

Cycles meeting a given strip form either " $L$ 's and $\Gamma$ 's" or "backwards $L$ 's and $\Gamma$ 's." This gives the directions: $L$ 's and $\Gamma$ 's read left to right!

We see from Figure 2 that the edges of a cycle are homotopic to three edges of $T, a, b, c$ say, cyclically repeated. The vertices are the same three edges of $T$ arranged so that if an edge is $a$, its vertices are $b$ and $c$ and so on (as in Figure 4). Thus for any (forwards or backwards) $L$ or $\Gamma$ : the edge at the down-stroke (a strip-crossing edge) equals the edge seen at the other end of its horizontal stroke. Further, the edge seen at any horizontal stroke equals the edge at the other end of the down-stroke. This proves the lemma.

3.4. Reduction to $T_{0}$. Let $G$ be the image of the holonomy representation of $\pi_{1}(M)$ in $\operatorname{Isom}\left(\mathbb{H}^{3}\right)$ so that $M=\mathbb{H}^{3} / G$ as usual. Let $x$ be a fixed point of some maximal parabolic subgroup of $G$ which we identify with the group of translations $G_{0}$ defined above. We regard symmetries and hidden symmetries as isometries of $\mathbb{H}^{3}$. Since $M$ is 1-cusped we can compose any symmetry or hidden symmetry with an element of $G$ to obtain an isometry which fixes $x$. Thus if $M$ has a symmetry, 
it is represented by a symmetry of $T_{0}$; if it has a non-trivial hidden symmetry, it is represented by a symmetry of $T_{0}$ which does not come from a symmetry of $M$.

Since our combinatorial picture of $T_{0}$ is not metrically exact, we only know that symmetries and hidden symmetries act as simplicial homeomorphisms (S.H.) of $T_{0}$.

Lemma 3.4. Simplicial homeomorphisms of $T_{0}$ preserve horizontal strips whenever $w \neq(L R)^{m}$ or $(L L R R)^{m}$ as a cyclic word, for any $m>0$.

Since vertex orders in $T_{0}$ are all even we can define a straight line to be a path in the 1-skeleton which enters and leaves each vertex along opposite edges. A strip consists of a part of $T_{0}$ between two (infinite, disjoint) straight lines such that every interior edge crosses from one side of the strip to the other.

Proof. If an edge of $T_{0}$ lies on the edge of a strip, not necessarily horizontal, the opposite vertex of the triangle which crosses that strip must have order at least 6 : the straight line going through this vertex has at least the two edges of the triangle on one side.

If there exists an S.H. which is not horizontal strip preserving, every vertex of $T_{0}$ will lie on a non-horizontal edge which is an edge of some strip (namely the image of a previously horizontal strip edge).

Suppose there is a vertex of order 10 or more, corresponding to 3 or more adjacent $L$ 's or $R$ 's in $w$ :

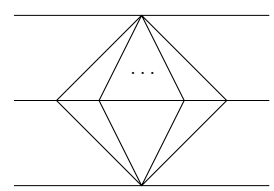

Here none of the non-horizontal edges shown can lie on a strip edge because they are all opposite vertices of order 4 . So in this case all S.H.'s must preserve horizontal strips.

Suppose there is a vertex of order 8 , corresponding to $L L$ or $R R$ in $w$ :

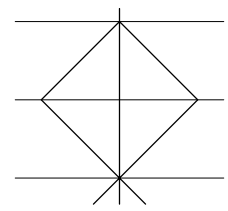

Now the only non-horizontal edges shown which can be strip edges are the vertical ones. Vertex orders on this vertical edge alternate $4,8,4,8$. So if an S.H. maps a horizontal strip edge to this vertical one, vertex orders along some horizontal edge must be $4,8,4,8, \ldots$. This determines $T_{0}$ and hence $w$ as $(L L R R)^{m}$. (The reader can verify that this particular $T_{0}$ admits a $\pi / 2$ rotation.)

Finally suppose there is no vertex of order $>6$. It follows immediately that $w$ is $(L R)^{m}$ and $T_{0}$ is the tiling of the plane by equilateral triangles. (Again, this admits S.H.'s which are not horizontal strip preserving.)

Lemma 3.5. Simplicial homeomorphisms of $T_{0}$ coming from symmetries of $T$ preserve the horizontal strip directions whenever $w \neq(L R)^{m}$ or $(L L R R)^{m}$.

Proof. An S.H. of $T_{0}$ which comes from a symmetry of $T$ obviously preserves the order of every edge of $T$. Thus if we label each edge of $T_{0}$ with the order of the corresponding edge of $T$, the labels must be preserved.

The order of an edge of $T$ corresponding to a vertex of $T_{0}$ is simply its order as a vertex of $T_{0}$. By Lemma 3.3 we can use the strip directions to label the 
corresponding edges of $T_{0}$. Given a vertex of order 10 or more we have:

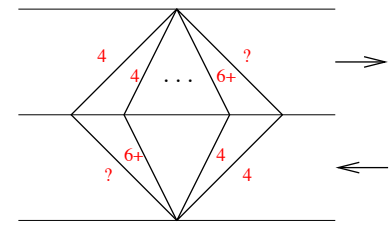

The direction on a horizontal strip cannot be reversed because this would swap an edge of order 4 with one of order 6 or more.

Suppose now the maximum vertex order is 8 . For each order 8 vertex we have:

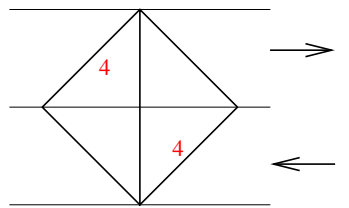

If there is an S.H. which reverses strip direction then the other two sides of this diamond figure, wherever it appears, must also be labelled with 4's.

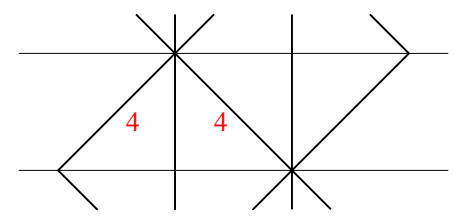

The 4 on the upper right edge implies that the right hand vertex of the diamond has order $\geq 8$, hence 8 . This gives another diamond figure adjacent to the first one. The argument can be repeated giving the pattern associated with $w=(L L R R)^{m}$.

If there are no vertices of order $>6, w=(L R)^{m}$.

Proof of Theorem 3.1. It is shown in [7] that the only arithmetic orientable hyperbolic punctured torus bundles are those for which $w=L R, L L R$ (or $L R R$ ) or $L L R R$, or powers of these, having invariant trace fields $\mathbb{Q}(\sqrt{-3}), \mathbb{Q}(\sqrt{-7})$ and $\mathbb{Q}(\sqrt{-1})$ respectively. Thus, in the non-arithmetic cases, Lemmas 3.4 and 3.5 show that all symmetries of $T_{0}$ which come from hidden symmetries of $M$ are represented by simplicial homeomorphism which preserve the strips and strip-directions.

Note that there are two "sister" manifolds for each $w$ depending on whether the monodromy is $\varphi_{w}$ or $-\varphi_{w}$; the triangulations of $\mathbb{H}^{3}$ however are the same, depending only on $w$.

It is now easy to see that the possible symmetries of $T_{0}$, modulo the translations in $G_{0}$, are restricted to the types listed below. We show that each, if it occurs at all, comes from an actual symmetry of $M$.

(1) Shifting up or down by two strips. This is realized by the symmetry $\left(\begin{array}{rr}-1 & 0 \\ 0 & -1\end{array}\right) \times 1$ from $F \times_{ \pm \varphi_{w}} S^{1}$ to itself. (Note that $\left(\begin{array}{rr}-1 & 0 \\ 0 & -1\end{array}\right)$ is central in $\operatorname{SL}(2, \mathbb{Z})$.)

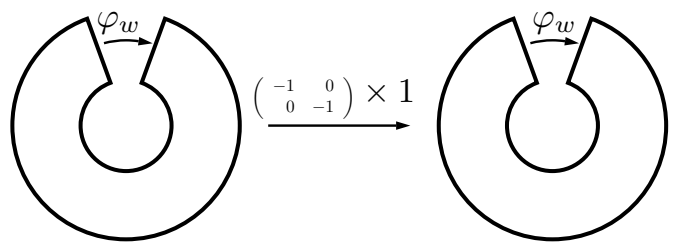


(2) If $w$ is a power, $w=u^{m}$, then $T_{0}$ admits a horizontal translation by $\ell(u)$ triangles, where $\ell(u)$ is the length of the word $u$. In this case

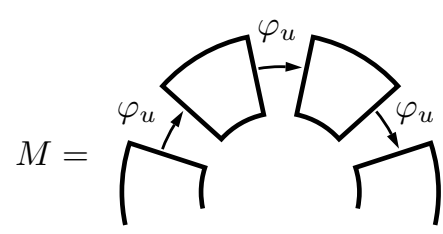

and $1 \times r_{2 \pi / m}$ gives a symmetry of order $m$ (where $r_{\theta}$ denotes rotation of the unit circle $S^{1}$ by $\theta$ ). For the $-\varphi_{w}$ case we replace one of the $\varphi_{u}$ 's by $-\varphi_{u}$. After rotating we also have to apply $-1 \times 1$ to $F \times[0,1 / m]$.

(3) If $w$ is palindromic, i.e. $w$ and its reverse $w^{\prime}$ are the same as cyclic words, then $T_{0}$ admits a rotation by $\pi$ about a point on one of the strip edges. This is realized by $\left(\begin{array}{rr}1 & 0 \\ 0 & -1\end{array}\right) \times \operatorname{refl}\left(S^{1}\right)$, where $\operatorname{refl}\left(S^{1}\right)$ denotes a reflection of $S^{1}$.

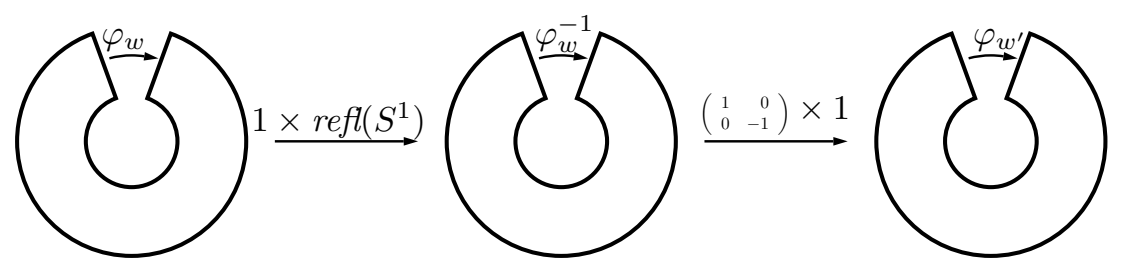

Note that conjugation by $\left(\begin{array}{rr}1 & 0 \\ 0 & -1\end{array}\right)$ takes $L$ to $L^{-1}$ and $R$ to $R^{-1}$.

(4) If $\ell(w)$ is even and rotating $w$ by a half turn swaps $L$ 's and $R$ 's, $T_{0}$ has a glide reflection mapping a strip to itself, exchanging the top and bottom of the strip. This is realized by $\left(\begin{array}{ll}0 & 1 \\ 1 & 0\end{array}\right) \times 1$ since conjugation by this matrix swaps $L$ and $R$. More explicitly, let $w=u v$ where $v$ is $u$ with $L$ 's and $R$ 's interchanged. The symmetry is

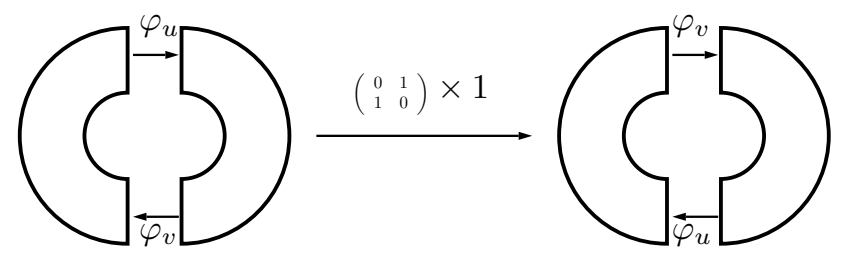

followed by $r_{\pi}$ on the $S^{1}$ factor. This symmetry is orientation reversing.

(5) If $\ell(w)$ is even and reversing $w$ swaps $L$ 's and $R$ 's (we might say that $w$ is anti-palindromic) then $T_{0}$ admits a glide reflection with vertical axis, shifting everything up by one strip. This is realized by $\left(\begin{array}{rr}0 & 1 \\ -1 & 0\end{array}\right) \times$ $\operatorname{refl}\left(S^{1}\right)$ since conjugation by $\left(\begin{array}{rr}0 & 1 \\ -1 & 0\end{array}\right)$ takes $L$ to $R^{-1}$ and $R$ to $L^{-1}$. This symmetry is orientation reversing. We leave the picture as an exercise for the reader.

Clearly if $M$ admits any two of the symmetries 3-5 it admits the third which is a product of the other two. Thus if $w$ is not a power, $M$ may have one of 5 possible symmetry groups. 


\section{Algorithm For Finding ISOMEtries of TILINGS}

To apply our commensurability criterion, Theorem 2.4 we need a way to determine when tilings of $\mathbb{H}^{n}$, arising from ideal cell decompositions, are isometric. We pursue this question in a slightly more general setting.

Let $M$ and $M^{\prime}$ be geometric $n$-manifolds with universal cover $X^{n}=\mathbb{E}^{n}$ or $\mathbb{H}^{n}$, each with a given finite decomposition into convex polyhedra. We wish to determine whether the tilings $T$ and $T^{\prime}$ which cover these two cell decompositions are isometric. (The elements of $T$ (resp. $T^{\prime}$ ) are convex polyhedra in $X^{n}$ which project to polyhedra in the decomposition of $M\left(\right.$ resp. $\left.M^{\prime}\right)$.)

Necessary and sufficient conditions for the tilings to be isometric are as follows.

(1) There is an isometry $i$ of $X^{n}$ which maps an element of $T$ isometrically onto an element of $T^{\prime}$.

(2) Whenever $i$ maps $P \in T$ isometrically onto $P^{\prime} \in T^{\prime}$, and $F$ is a (codimension 1) face of $P, i$ maps the neighbour of $P$ at $F$ isometrically onto the neighbour of $P^{\prime}$ at $i(F)$.

Sufficiency follows from the fact that we can proceed from any tile in $T$ to any other by a finite sequence of steps between neighbouring tiles.

Let $S$ and $S^{\prime}$ denote the polyhedra decomposing $M$ and $M^{\prime}$ respectively.

Let $\Theta$ denote the set of all triples $\left(j, p, p^{\prime}\right)$, where $p \in S, p^{\prime} \in S^{\prime}$, and $j$ is an isometry carrying $p$ onto $p^{\prime}$. We can find $\Theta$ in a finite number of steps. Condition 1 above is equivalent to $\Theta$ being non-empty.

We say that $\left(j, p, p^{\prime}\right) \in \Theta$ is induced by an isometry $i$ of $X^{n}$ if there exist $P \in T$ projecting to $p$ and $P^{\prime} \in T^{\prime}$ projecting to $p^{\prime}$ such that $i$ carries $P$ isometrically onto $P^{\prime}$ and the restriction induces $j$. Let $f$ be a face of $p$ and let $q$ and $q^{\prime}$ be the neighbours of $p$ and $p^{\prime}$ at $f$ and $j(f)$ respectively. The restriction of $j$ to $f$ induces an isometry between certain faces of $q$ and $q^{\prime}$. If this extends to $\left(j_{q}, q, q^{\prime}\right)$ we say that $\left(j, p, p^{\prime}\right)$ extends across $f$ to $\left(j_{q}, q, q^{\prime}\right)$. If $\left(j, p, p^{\prime}\right)$ is induced by $i$ then $\left(j_{q}, q, q^{\prime}\right)$ will be also.

Condition 2 is equivalent to the following: whenever $\left(j, p, p^{\prime}\right)$ is induced by $i$, and $f$ is a face of $p$, then $\left(j, p, p^{\prime}\right)$ extends across $f$.

Theorem 4.1. With the above notation, the tilings $T$ and $T^{\prime}$ are isometric if and only if there exists a non-empty subset $I$ of $\Theta$ such that every element of $I$ extends across each of its faces to yield another element of $I$.

Proof. If $T$ and $T^{\prime}$ are isometric with isometry $i$, simply let $I$ be the set of elements of $\Theta$ induced by $i$.

Conversely, suppose we have $I \subseteq \Theta$ having the stated properties. Choose any element $\left(j, p, p^{\prime}\right) \in I$ and any isometry $i$ of $X^{n}$ which induces it. Clearly condition 1 above is satisfied. For condition 2 note that if $i$ maps $P$ onto $P^{\prime}$ and this induces $\left(j, p, p^{\prime}\right) \in I$ then, because this extends across all of its faces, $i$ maps neighbours of $P$ isometrically onto neighbours of $P^{\prime}$ and these too induce elements of $I$. Therefore condition 2 is satisfied with all the induced triples belonging to $I$.

An algorithm for finding such a subset $I \subseteq \Theta$ or establishing that none exists is straightforward. If $\Theta$ is empty, stop; there is no such subset. Otherwise choose any element of $\Theta$ and put it in $I$. For each element of $I$ check if we can extend across all faces. If we can't, remove all elements of $I$ from $\Theta$ and start again. If we can, and every element we reach is already in $I$, stop and output $I$. If we can extend across faces of all elements of $I$, and we obtain new elements not yet in $I$, add those new elements to $I$ and check again.

Each set $I$ found by the algorithm represents an equivalence class of isometries carrying $T$ onto $T^{\prime}$ : choose an isometry $i$ inducing any element of $I$; then isometries 
$i, i^{\prime}$ are equivalent if $i^{\prime}=g^{\prime} i g$ where $g$ is a covering transformation of $M$ and $g^{\prime}$ is a covering transformation of $M^{\prime}$.

To find the symmetry group of a tiling $T$ we apply the algorithm with $T=T^{\prime}$, $M=M^{\prime}$ etc. Let $\Gamma$ denote the group of covering transformations of $M$. Repeating the algorithm until $\Theta$ is exhausted we obtain finitely many equivalence classes of symmetries. These represent the double cosets of $\Gamma$ in $\operatorname{Symm}(T)$. Together with $\Gamma$ they generate $\operatorname{Symm}(T)$.

Remark 4.2. Each set I found by this algorithm also represents a common covering $N$ of $M$ and $M^{\prime}$ constructed as follows: For each element $r=\left(j, p, p^{\prime}\right) \in I$ let $p_{r}$ denote a copy of $p$. Let $N$ be the disjoint union of the polyhedra $p_{r}$ as $r$ varies over $I$, with the following face identifications. Each face $f$ of $p$ appears as a face $f_{r}$ of $p_{r}$. Whenever $r$ extends across $f$ to $s=\left(j_{q}, q, q^{\prime}\right)$, identify face $f_{r}$ of $p_{r}$ with face $f_{s}$ of $q_{s}$.

4.1. Example. Let $M$ be the Euclidean torus obtained by gluing a 1 by 3 rectangle along opposite pairs of edges. Let $M^{\prime}$ be another such torus obtained by gluing a 2 by 1 rectangle. Subdivide each into unit squares as shown, so that $S$, the subdivision of $M$, equals $\{A, B, C\}$ while $S^{\prime}=\{P, Q\}$. Both $S$ and $S^{\prime}$ lift to tilings of the plane by unit squares.
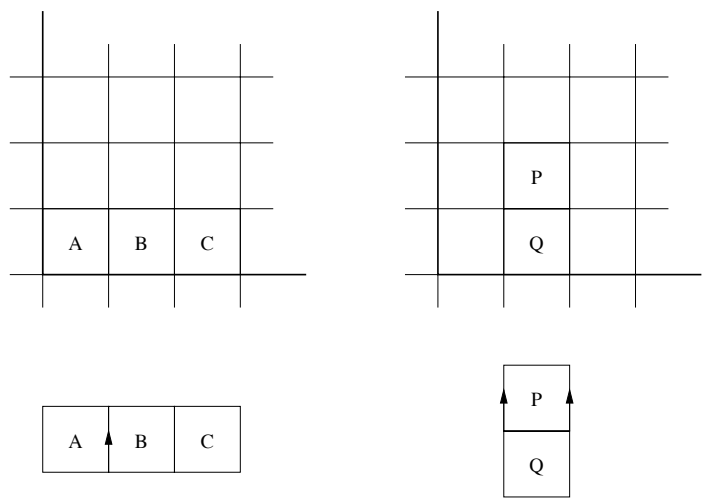

$\Theta$ consists of 48 elements since there are 8 ways of isometrically mapping one unit square onto another and 6 combinations of squares to be mapped. Suppose we start with $\left(t_{A P}, A, P\right)$ where $t_{A P}$ denotes the translation carrying $A$ onto $P$ in the picture. We can extend $\left(t_{A P}, A, P\right)$ across the marked edge (whose image in $M^{\prime}$ is also shown) to obtain $\left(t_{B P}, B, P\right)$. Continuing until we have extended across every available edge we obtain 6 elements of $\Theta$ which we can abbreviate to $\left\{t_{A P}, t_{B P}, t_{C P}, t_{A Q}, t_{B Q}, t_{C Q}\right\}$. These give rise to the following common covering of $M$ and $M^{\prime}$.

\begin{tabular}{|c|c|c|}
\hline${ }^{t}{ }_{A P}$ & $t_{B P}$ & $\mathrm{t}_{\mathrm{CP}}$ \\
\hline${ }^{\mathrm{t}} \mathrm{AQ}$ & ${ }^{\mathrm{t}} \mathrm{BQ}$ & ${ }^{\mathrm{t}} \mathrm{CQ}$ \\
\hline
\end{tabular}

Suppose instead we begin with a clockwise rotation $r_{A P}$ through $90^{\circ}$ carrying $A$ onto $P$. Then we obtain a different common cover.

\begin{tabular}{|l|l|l|l|l|l|}
\hline${ }_{\mathrm{AP}}$ & ${ }_{\mathrm{BQ}}$ & ${ }_{\mathrm{CP}}$ & ${ }_{\mathrm{AQ}}$ & ${ }_{\mathrm{BP}}$ & ${ }^{\mathrm{r}} \mathrm{CQ}$ \\
\hline
\end{tabular}


Suppose finally we subdivide one of the squares in $S^{\prime}$ into two triangles. Now the tilings are clearly not isometric.
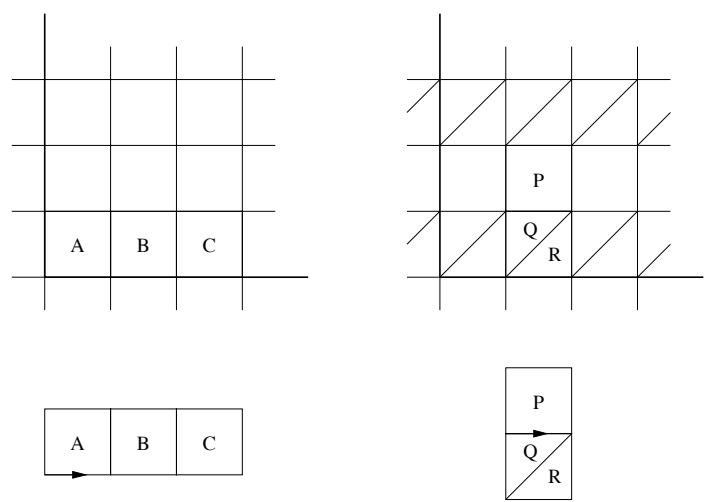

The algorithm might start with $t_{A P}$ but when it tries to extend this across the marked edge it will find that the induced mapping from an edge of $A$ to an edge of $Q$ does not extend to any isometry from $A$ to $Q$.

4.2. Combinatorial construction of coverings. Mostow-Prasad rigidity often allows us to work in a purely combinatorial setting; this motivates the following. The algorithm described above can be viewed as a method for constructing a common cover of two manifolds which admit suitable subdivisions into polyhedra. The algorithm works equally well for topological spaces $M$ and $M^{\prime}$ admitting $n$-dimensional polyhedral decompositions (i.e. obtained by gluing $n$-dimensional, Euclidean or finite volume hyperbolic, convex polyhedra pairwise along their $(n-1)$ dimensional faces, such that open faces of dimension $q \leq n$ embed). We then let each element $\left(j, p, p^{\prime}\right) \in \Theta$ represent a combinatorial isomorphism between $p$ and $p^{\prime}$. (We can barycentrically subdivide the decompositions of $M$ and $M^{\prime}$ in order to realize the $j$ 's as piecewise linear homeomorphisms in a canonical way.) Now, if the algorithm is successful it constructs, in general, a branched covering of $M$ and $M^{\prime}$, branched over the codimension 2 skeleta of $M$ and $M^{\prime}$.

When $M$ and $M^{\prime}$ are PL-manifolds, we would like to construct an unbranched cover which is also a manifold. It is sufficient to check that the cover we construct does not branch over any codimension 2 cell of $M$ or $M^{\prime}$. For if this is the case we proceed by induction on $q$ to show that our cover is not branched over any codimension $q$ cell of $M$ (or $M^{\prime}$ ). By construction, there is no branching over cells of codimension 0 and 1 , and we assume that there is no branching over cells of codimension 2. So suppose $q \geq 3$ and there is no branching over cells of codimension $\leq q-1$. Then the link of a codimension $q$ cell in the cover is a connected unbranched covering of the link of a codimension $q$ cell of $M$. But since the latter is a $(q-1)$ sphere, hence simply connected since $q \geq 3$, the covering is a homeomorphism.

In the case of most interest to us, namely when $M$ and $M^{\prime}$ are cusped hyperbolic 3-manifolds, Mostow-Prasad rigidity ensures that if $N$ topologically covers both $M$ and $M^{\prime}$ then the induced hyperbolic structures on $N$ are isometric.

Frequently, an ideal cell decomposition of a hyperbolic 3-manifold consists entirely of ideal tetrahedra. If this is the case for $M$ and $M^{\prime}$, then our algorithm will always find a common cover, branching over the edge sets of the two manifolds (elements of $\Theta$ will always extend over their faces because all tetrahedra are combinatorially equivalent). This will indicate that the two manifolds are commensurable only if the covering is in fact unbranched. When an element of $\Theta$ induces a mapping of an edge $e$ of (the ideal cell decomposition of) $M$ onto an edge $e^{\prime}$ of $M^{\prime}$, the resulting cover will be unbranched if and only if the order of $e$ (the number of 
tetrahedra to which glue around it) is equal to the order of $e^{\prime}$. For example, this has the following corollary.

Theorem 4.3. If a finite volume hyperbolic 3-manifold $M$ admits a decomposition into ideal tetrahedra such that the order of every edge is 6 , then $M$ is commensurable with the complement of the figure-8 knot.

\section{ENUMERATING CANONICAL CELL DECOMPOSITIONS I}

For 1-cusped hyperbolic manifolds with discrete commensurator we now have all the ingredients of an effective method for testing commensurability. For multicusped manifolds we need a way to search through the (finite) set of all canonical ideal cell decompositions. In this section and the next we describe two alternative approaches to this problem, while in Section 7 we show how the search can be restricted for greater efficiency.

The first approach is very simple minded: if we can bound the degree with which $M$ covers its commensurator quotient, we can enumerate all cusp cross sections which could possibly cover equal area cross sections in the quotient. Such degree bounds can be obtained from estimates on the the minimum volume of cusped non-arithmetic hyperbolic 3-manifolds ([26, [1], [28]).

The second approach is geometric and may be of more theoretical interest since it truly finds all the canonical cell decompositions. In fact it associates with a given $c$-cusped $M$, a convex polytope in $\mathbb{R}^{c}$ whose $k$-dimensional faces, for $0 \leq k<c$, are in 1-1 correspondence with canonical cell decompositions of $M$.

Let $M$ be a hyperbolic orbifold with (horoball) cusp neighbourhoods $C_{1}, \ldots, C_{m}$ and let $N$ be a degree $d$ quotient of $M$ with corresponding cusp neighbourhoods $c_{1}, \ldots, c_{n}$. Let $\pi: M \rightarrow N$ be the covering projection and let $c_{j(i)}=\pi\left(C_{i}\right)$. If we choose horospherical cross sections in $N$ with "area" (i.e. codimension 1 volume) equal to 1 , the area of $\partial C_{i}$ is equal to the degree with which $C_{i} \operatorname{covers} c_{j(i)}$. The sum of the areas of the $C_{i}$ covering $c_{j}$ will be $d$ for each $c_{j}$.

Thus, in order to find a possible degree $d$ quotient of $M$ via the canonical cell decomposition, we can enumerate the possible integer area vectors as follows. For each $n, 1 \leq n \leq m$, and each partition of $\{1, \ldots, m\}$ into $n$ non-empty subsets $I_{1}, \ldots, I_{n}$, enumerate all area vectors $\left(a_{1}, \ldots, a_{m}\right)$ such that each $a_{i}$ is a positive integer and $\sum_{i \in I_{j}} a_{i}=d$ for $j=1, \ldots, n$.

Example: if $m=3$ we have partitions $\{\{1,2,3\}\},\{\{1,2\},\{3\}\},\{\{1,3\},\{2\}\}$, $\{\{1\},\{2,3\}\}$ and $\{\{1\},\{2\},\{3\}\}$. The first partition admits $\frac{1}{2}(d-1)(d-2)$ area vectors, the next three admit $d-1$ each, and the last, just one.

We can enumerate area vectors corresponding to possible quotients of degree $d$ as follows. Any quotient of $M$ of degree $d$ has $n \leq m$ cusps and has a corresponding area vector $\left(a_{1}, \ldots, a_{m}\right)$ with sum $\sum_{i=1}^{m} a_{i}=n d$. So if we fix an integer $D \geq m$ and enumerate all positive integer area vectors summing to at most $D$, we will find all quotients of degree $d$ with $n \leq m$ cusps such that $n d \leq D$. In particular this will include all quotients with at most $m-1$ cusps provided $d \leq D /(m-1)$. Since the canonical cell decomposition is determined by the ratio of the areas, any $m$ cusped quotient is found using the area vector $(1, \ldots, 1)$ with $\sum_{i} a_{i}=m \leq D$. So we will find all canonical cell decompositions arising from quotients of degree $d \leq D /(m-1)$.

\section{ENUMERATING CANONICAL CELL DECOMPOSITIONS II}

Let $M$ be a hyperbolic $n$-manifold with $c>0$ cusps. Then the set of possible choices of (not necessarily disjoint) horospherical cross sections dual to the cusps of $M$ is parametrized by the vector of their areas (i.e. $(n-1)$-dimensional volumes) 
in $\mathbb{R}_{>0}^{c}=\left\{\left(v_{1}, \ldots, v_{c}\right) \in \mathbb{R}^{c} \mid v_{1}>0, \ldots, v_{c}>0\right\}$. In fact it turns out to be more convenient to parametrize by the $(n-1)$ th root of area, a quantity we will refer to as size. Multiplying a size vector $v \in \mathbb{R}_{>0}^{c}$ by a constant $\lambda>0$ has the effect of shifting the corresponding horospherical cross sections a distance $\log (\lambda)$ down (i.e. away from) the cusps.

For each $v \in \mathbb{R}_{>0}^{c}$ we obtain a Ford spine. It is clear from the definition that if we choose a set of disjoint horospherical cross sections and then shift them all up or down by the same amount we get the same Ford spine. Thus the set of possible Ford spines is parametrized by the set of rays in $\mathbb{R}_{>0}^{c}$, or equivalently, by points in the open $(c-1)$-dimensional simplex $\mathcal{S}=\left\{\left(v_{1}, \ldots, v_{c}\right) \in \mathbb{R}_{>0}^{c} \mid v_{1}+\cdots+v_{c}=1\right\}$. For a 1-cusped manifold the Ford spine is unique.

Dual to each Ford spine is a canonical cell decomposition $D(v)$. As we vary $v \in \mathcal{S}$ the decomposition changes only when the combinatorics of the spine changes. To better understand this dependence we now review an alternative approach to defining $D(v)$, namely the original one of Epstein-Penner in [11.

We work in Minkowski space $\mathbb{E}^{n, 1}$ with the inner product $*$ defined by

$$
x * y=x_{1} y_{1}+\ldots+x_{n} y_{n}-x_{n+1} y_{n+1}
$$

for $x=\left(x_{1}, \ldots, x_{n+1}\right), y=\left(y_{1}, \ldots, y_{n+1}\right)$ in $\mathbb{R}^{n+1}$. Then hyperbolic space $\mathbb{H}^{n}$ is the upper sheet of the hyperboloid $x * x=-1$, and the horospheres in $\mathbb{H}^{n}$ are represented by the intersections of hyperplanes, having light-like normal vectors, with $\mathbb{H}^{n}$. Each such hyperplane $H$ has a unique Minkowski normal $\mathbf{n}$ such that $x \in H$ if and only if $x * \mathbf{n}=-1$.

Let $\Gamma$ denote the group of covering transformations of $\mathbb{H}^{n}$ over $M$. Each size vector $v$ gives rise to a $\Gamma$-invariant set of horospheres in $\mathbb{H}^{n}$. The resulting set of normals in Minkowski space is invariant under the action of the group $\Gamma$. The convex hull of this set of points, which we shall refer to as the Epstein-Penner convex hull, intersects every ray based at the origin passing through a point in the upper sheet of the hyperboloid. The boundary of this convex set is a union of closed convex $n$-dimensional polytopes having coplanar light-like vertices. Epstein and Penner [1] show that these project to a locally finite, $\Gamma$-invariant set of ideal polyhedra in $\mathbb{H}^{n}$, which in turn project to a finite set $D(v)$ of ideal hyperbolic polyhedra in $M$.

Starting with a given ideal hyperbolic cell decomposition $D$ of $M$ and a size vector $v$, we proceed next to describe necessary and sufficient conditions for $D$ to be the canonical cell decomposition $D(v)$, as in 33 and 30 .

Let $C$ be a cell of $D$. Then $v$ determines a horospherical cross section to each ideal vertex of $C$. We lift $C$ and this choice of horospheres to $\mathbb{H}^{n}$. In Minkowski space, this gives a convex (Euclidean) $n$-dimensional polytope whose vertices are the hyperplane normals for these horospheres. Whenever $C$ is not a simplex, it is necessary to add the condition that these vertices are coplanar. If this is satisfied for all the non-simplicial cells of $D$, we can lift each cell to an $n$-dimensional polytope in Minkowski space with vertices corresponding to the choice of horospheres determined by the size vector $v$. If $C$ and $C^{\prime}$ are neighbouring cells in $D$ it is necessary that the angle between neighbouring lifts into Minkowski space be convex upwards. Together these conditions are also sufficient to imply $D=D(v)$.

These conditions can be expressed as a set of linear equations and linear inequalities on the entries of the size vector $v$.

Proposition 6.1. Let $D$ be an ideal hyperbolic cell decomposition of a cusped hyperbolic $n$-manifold $M$ with c cusps. Then there exist matrices $L_{D}$ and $F_{D}$ with $c$ columns such that, for $v \in \mathbb{R}_{>0}^{c}, D(v)=D$ if and only if $L_{D} v=0$ and $F_{D} v>0$. 
Note: here $v$ is written as a column vector, and the condition $F_{D} v>0$ means that each entry of the vector $F_{D} v$ is positive.

Proof. Let $v_{i}$ denote the entry of $v$ corresponding to the $i$ th cusp of $M$. Let $\mathbf{n}_{j}$ be the vertex representative for the $j$ th vertex of $C$ lifted to Minkowski space for the choice of horospheres given by $v=(1, \ldots, 1)$. Then for an arbitrary size vector $v$ the corresponding representative is $\mathbf{n}_{j} / v_{c(j)}$, where $c(j)$ is the cusp of the $j$ th vertex of $C$.

The coplanarity condition on a non-simplicial cell $C$ gives a set of linear equations satisfied by $v$, one for each vertex of $C$ in excess of $n+1$, as follows. Let $N_{v}$ be a Euclidean normal to the hyperplane containing $\left\{\mathbf{n}_{0} / v_{c(0)}, \ldots, \mathbf{n}_{n} / v_{c(n)}\right\}$ such that $\left(\mathbf{n}_{j} / v_{c(j)}\right) \cdot N_{v}=1$ for $j=0, \ldots, n$, where $\cdot$ denotes the Euclidean dot product. Writing $M_{C}$ for the inverse of the matrix with rows $\mathbf{n}_{j}$ we obtain $N_{v}=M_{C}\left(v_{c(0)}, \ldots, v_{c(n)}\right)^{t}$, which is a linear function of $v$. For $j>n, \mathbf{n}_{j} / v_{c(j)}$ belongs to this hyperplane if and only if $\mathbf{n}_{j} \cdot N_{v}-v_{c(j)}=0$, which is linear in $v$. The full set of constraints for $C$ gives a matrix equation $L_{C} v=0$.

The convexity condition at an $(n-1)$-cell $f$ of $D$, being the common face of $n$-cells $C$ and $C^{\prime}$, can be expressed as follows. Let $N_{v}$, as above, be the defining normal for the hyperplane containing the lift of $C$ determined by $v$. Let $\mathbf{n}_{k}^{\prime} / v_{c(k)}$ be a vertex of an adjacent lift of $C^{\prime}$, not in the lift of $f$. This vertex lies above the hyperplane if and only if $\left(\mathbf{n}_{k}^{\prime} / v_{c(k)}\right) \cdot N_{v}>1$, or equivalently $\mathbf{n}_{k}^{\prime} \cdot N_{v}-v_{c(k)}>0$. We refer to the left-hand side of this inequality as the tilt at $f$ of $v$ and express the condition as $F_{f} v>0$, where $F_{f}$ is a suitable row-vector. (Note that the sign of our tilt function is opposite to that of [33] and 31.)

Finally, concatenate the matrices $L_{C}$ into a matrix $L_{D}$ and the rows $F_{f}$ into a matrix $F_{D}$.

Let $\mathcal{P}_{D}$ denote the set of $v \in \mathbb{R}_{>0}^{c}$ such that $L_{D} v=0$ and $F_{D} v>0$. We call this the parameter cell of $D$ since it contains all cusp size parameters $v$ such that $D(v)=D$. Each $v \in \mathbb{R}_{>0}^{c}$ belongs to a parameter cell, namely $\mathcal{P}(v)=\mathcal{P}_{D(v)}$. The parameter cell $\mathcal{P}_{D}$ is non-empty if and only if $D$ is a canonical cell decomposition.

It is shown in 44 that the number of canonical cell decompositions is finite. Therefore $\mathbb{R}_{>0}^{c}$ is a union of finitely many parameter cells.

Proposition 6.2. Each $v \in \mathbb{R}_{>0}^{c}$ can be perturbed to obtain a nearby vector $v^{\prime}$ such that $\mathcal{P}\left(v^{\prime}\right)$ has dimension $c$ and $\mathcal{P}(v)$ is a face of $\mathcal{P}\left(v^{\prime}\right)$ (or equals $\mathcal{P}(v)$ if this has dimension $c$ ).

Proof. Let $D=D(v)$. If $L_{D}$ is zero (or empty) then $\mathcal{P}(v)$ is an open subset, hence a $c$-dimensional cell and we just set $v^{\prime}=v$.

Otherwise, perturb $v$ such that it leaves the linear subspace determined by $L_{D} v=$ 0. For a small perturbation the Epstein-Penner convex hull changes as follows: no dihedral angle between adjacent $n$-faces goes to $\pi$ but some non-simplicial $n$-faces may be subdivided if their vertices become non-coplanar. It follows that $L_{D}$ may lose rows and $F_{D}$ may gain rows. Let $D^{\prime}$ be the new decomposition. Then $L_{D^{\prime}} \neq L_{D}$ because $L_{D^{\prime}} v^{\prime}=0$ while $L_{D} v^{\prime} \neq 0$. Repeat until $L_{D^{\prime}}$ is zero (or empty). Then $\mathcal{P}\left(v^{\prime}\right)$ has dimension $c$.

Now $v$ belongs to a face of $\mathcal{P}_{D^{\prime}}$ since it satisfies $F_{f} v>0$ for each face $f$ common to $D$ and $D^{\prime}$, and $F_{f^{\prime}} v=0$ for each face $f^{\prime}$ of $D^{\prime}$ not in $D$. The former condition amounts to $F_{D} v>0$. We have to show that the latter is equivalent to $L_{D} v=0$. But that is equivalent to the coplanarity of the lifted vertices of each $n$-cell of $D$. Such a cell may be subdivided by new faces $f^{\prime}$ in $D^{\prime}$. Then the vertices will be coplanar at $v$ if and only if the tilt at each subdividing face is zero, i.e. if and only if $F_{f^{\prime}} v=0$ for the subdividing faces. 
The above proposition implies that each face of a parameter cell is another parameter cell; the decomposition corresponding to a parameter cell is a refinement of the decompositions corresponding to its faces.

Remark 6.3. It is tempting to suppose that the canonical cell decomposition of $M$ corresponding to a $c$ dimensional parameter cell must consist entirely of ideal simplices but this need not be the case. In general we can have cell decompositions with non-simplicial cells such that $L_{D}$ is a zero matrix. (For example, this occurs for the Borromean rings complement - see Section 6.1 below.)

We now have the following algorithm for finding all canonical cell decompositions. First we find all the $c$-dimensional parameter cells.

(1) Choose an arbitrary $v \in \mathbb{R}_{>0}^{c}$.

(2) Perturb $v$ if necessary, as in the proof of Proposition 6.2, so that $\mathcal{P}(v)$ has dimension $c$, and add it to our list of cells.

(3) If the closure of the cells we have found so far does not contain the whole of $\mathbb{R}_{>0}^{c}$, choose a new $v$ not in the closure of any cell found so far and repeat step 2 .

By the finiteness result quoted above, this algorithm eventually terminates. We can then enumerate all canonical cell decompositions by enumerating the faces of all dimensions of the cells $\mathcal{P}(v)$.

While the computational geometry involved in implementing the above algorithm is certainly possible, it is not particularly nice. We explain a refinement which gives a little more insight and an algorithm which is easier to implement.

For a decomposition $D$ of $M$, let $\Sigma_{D}$ denote the row vector obtained by adding together the rows of $F_{D}$. We define the tilt polytope of $M$ to be the set of $v \in \mathbb{R}_{>0}^{c}$ such that $\Sigma_{D} \cdot v<1$ for all canonical cell decompositions $D$ of $M$.

Proposition 6.4. The tilt polytope $T$ of $M$ is bounded. The parameter cells $\mathcal{P}_{D}$ of $M$ are the cones over the origin of those faces of $T$ which are not contained in $\partial \mathbb{R}_{>0}^{c}$.

Proof. We show that the closure of a $c$-dimensional parameter cell $\overline{\mathcal{P}}_{D}$ has bounded intersection with $T$. Let $v$ be a unit vector in $\overline{\mathcal{P}}_{D}$. Then since $v$ is not contained in every face of $\mathcal{P}_{D}, \Sigma_{D} \cdot v>0$. The length of any multiple of $v$ contained in $T$ is bounded by $1 /\left(\Sigma_{D} \cdot v\right)$. Since this is continuous in $v$, and the set of such $v$ is compact, $\overline{\mathcal{P}}_{D} \cap T$ is bounded. Since $T$ is a union of finitely many such sets it is bounded.

Let us write $H_{D}$ for the half-space $\left\{x \in \mathbb{R}^{c} \mid \Sigma_{D} \cdot x<1\right\}$. Then $T$ is the intersection of all the $H_{D}$ 's with $\mathbb{R}_{>0}^{c}$. We will show that: if $v$ belongs to a $c$ dimensional parameter cell $\mathcal{P}_{D}$, and $\mathcal{P}_{D^{\prime}}$ is any other parameter cell, then the ray generated by $v$ leaves $H_{D}$ before it leaves $H_{D^{\prime}}$.

It will then follow that a ray in $\mathcal{P}_{D}$ penetrates the (non-empty) face of $T$ generated by $H_{D}$. Since a ray not in $\mathcal{P}_{D}$ belongs to the closure of some other parameter cell, it does not leave $H_{D}$ first and therefore does not pass through the same face of $T$. Since cones on the lower dimensional faces of a $(c-1)$-dimensional face of $T$ are the faces of a $c$-dimensional parameter cell, the result then follows from Proposition 6.2.

It remains to show that $H_{D}$ cuts off any ray in $\mathcal{P}_{D}$ closer to the origin than $H_{D^{\prime}}$, for all parameter cells $\mathcal{P}_{D^{\prime}} \neq \mathcal{P}_{D}$. Equivalently, for $v \in \mathcal{P}_{D}, \Sigma_{D} \cdot v>\Sigma_{D^{\prime}} \cdot v$.

Firstly, let $\mathcal{P}_{D}$ and $\mathcal{P}_{D^{\prime}}$ be any two parameter cells such that $\mathcal{P}_{D^{\prime}}$ is a face of $\mathcal{P}_{D}$, and let $v$ belong to $\mathcal{P}_{D}$. The rows of $F_{D^{\prime}}$ are a proper subset of the rows of $F_{D}$, and since $F_{f} \cdot v>0$ for each row, $\Sigma_{D} \cdot v>\Sigma_{D^{\prime}} \cdot v$. If instead $\mathcal{P}_{D}$ is a face 
of $\mathcal{P}_{D^{\prime}}$, then the rows of $F_{D^{\prime}}$ omitted from $F_{D}$ are precisely those for which $F_{f} \cdot v$ vanishes. Therefore in that case $\Sigma_{D} \cdot v=\Sigma_{D^{\prime}} \cdot v$.

Next, let $\mathcal{P}_{D}$ be a $c$-dimensional parameter cell, and let $\mathcal{P}_{D^{\prime}}$ be arbitrary, with $D^{\prime} \neq D$. Choose $v \in \mathcal{P}_{D}$ and $v^{\prime} \in \mathcal{P}_{D^{\prime}}$. Let $\mathcal{P}_{D_{1}}, \ldots, \mathcal{P}_{D_{m}}$ be the parameter cells through which the straight line $v_{t}:=(1-t) v+t v^{\prime}$ passes for $0 \leq t \leq 1$ (so that $D_{1}=D$ and $\left.D_{m}=D^{\prime}\right)$. For $v_{t}$ in $\mathcal{P}_{D_{i}}, \Sigma_{D_{i}} \cdot v_{t} \geq \Sigma_{D_{i+1}} \cdot v_{t}$ while for $v_{t} \in \mathcal{P}_{D_{i+1}}$, $\Sigma_{D_{i}} \cdot v_{t} \leq \Sigma_{D_{i+1}} \cdot v_{t}$. Since the difference between these terms is (affine) linear in $t$, the former inequality must hold for all lesser values of $t$, in particular, when $v_{t}=v$. Note also that the first such inequality is strict, namely, $\Sigma_{D_{1}} \cdot v>\Sigma_{D_{2}} \cdot v$. It follows that $\Sigma_{D} \cdot v>\Sigma_{D^{\prime}} \cdot v$ for arbitrary $D^{\prime}$.

Let $T_{0}$ be a polytope resulting from the intersection of $\mathbb{R}_{>0}^{c}$ with some of the half-spaces $H_{D}$ defined in the above proof. If $T_{0} \supsetneq T$, some face $A=\bar{T}_{0} \cap \partial H_{D}$ of $T_{0}$ will contain a point $v \in \mathbb{R}_{>0}^{c}$ not in $\bar{T}$, and thus not in $\bar{T} \cap \partial H_{D}$, nor in the cone on this, $\overline{\mathcal{P}}_{D}$. Therefore $L_{D} v \neq 0$ or $F_{f} v<0$ for some row $F_{f}$ of $F_{D}$. This gives a test for when $T_{0}$ properly contains $T$; when satisfied, it yields a new halfspace $H_{D(v)}$ whose intersection with $T_{0}$ is strictly smaller. After a finite number of intersections we arrive at $T_{0}=T$. See Figure 5 .

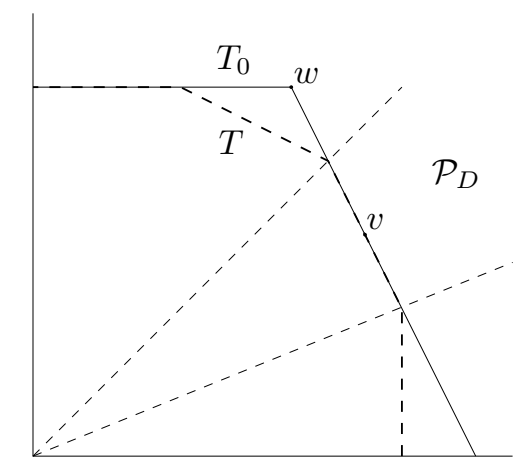

FiguRE 5. $T_{0}$ is a partially computed tilt polytope. The face of $T_{0}$ containing $v \in \mathcal{P}_{D}$ has a vertex $w$ not in $\overline{\mathcal{P}}_{D}$. Therefore $D(w)$ gives another face of $T$.

The computational geometry involved in the above is relatively straightforward. By using homogeneous coordinates we can treat an unbounded region, such as $\mathbb{R}_{>0}^{c}$, as a polytope with some vertices "at infinity". The face $A$ of $T_{0}$, as defined above, is the convex hull of those vertices $v$ of $T_{0}$ satisfying $\Sigma_{D} \cdot v=1$. If any of these satisfy $L_{D} v \neq 0$ or $F_{f} v<0$ for some row $F_{f}$ of $F_{D}$ we conclude that $T_{0} \neq T$. If such $v$ lies in $\partial \mathbb{R}_{>0}^{c}$ we perturb it a little to bring it inside $\mathbb{R}_{>0}^{c}$ before determining a new half-space $H_{D(v)}$.

6.1. Example: The Borromean rings complement. Let $M$ be the complement of the Borromean rings in $S^{3}$ :

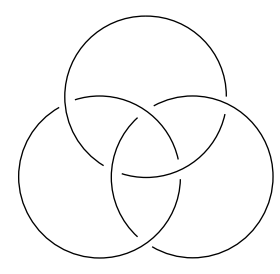


Then $M$ is an arithmetic hyperbolic 3-manifold with 3 cusps. It may be realized by gluing two regular ideal hyperbolic octahedra in the following pattern (see [32]).
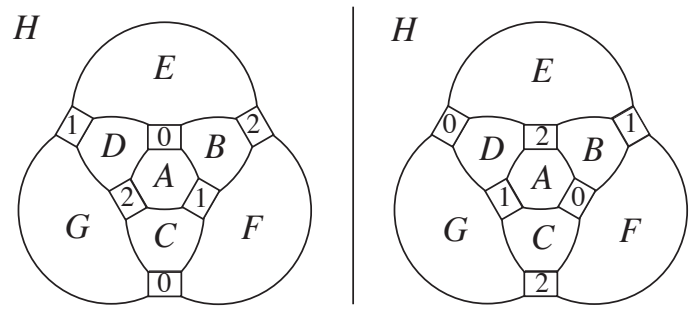

Letters indicate face identifications; cusps are numbered 0,1,2. Equal area cusp cross sections give rise to symmetrically placed ideal vertex cross sections in the two octahedra. It follows that the corresponding ideal cell decomposition consists of precisely these two octahedra. We will first compute its parameter cell.

Let us position the tiling of $\mathbb{H}^{3}$ by ideal octahedra such that one of them, call it $C$, has a lift with vertices at $\{( \pm 1,0,0),(0, \pm 1,0),(0,0, \pm 1)\}$ in the projective ball model. When the cusp cross sections all have equal area and contain the centre of the octahedron, their Minkowski normals are $\mathbf{n}_{0}=(1,0,0,1), \mathbf{n}_{1}=(0,1,0,1), \mathbf{n}_{2}=$ $(0,0,1,1), \mathbf{n}_{3}=(-1,0,0,1), \mathbf{n}_{4}=(0,-1,0,1), \mathbf{n}_{5}=(0,0,-1,1)$. A neighbouring lift of the other octahedron, call it $C^{\prime}$, has vertex representatives $\left\{\mathbf{n}_{0}, \mathbf{n}_{1}, \mathbf{n}_{2}, \mathbf{n}_{3}^{\prime}, \mathbf{n}_{4}^{\prime}, \mathbf{n}_{5}^{\prime}\right\}$ where $\mathbf{n}_{i}^{\prime}$ is the Minkowski metric reflection of $\mathbf{n}_{i}$ in the hyperplane spanned by $\left\{\mathbf{n}_{0}, \mathbf{n}_{1}, \mathbf{n}_{2}\right\}$. Then $\mathbf{n}_{3}^{\prime}=(1,2,2,3), \mathbf{n}_{4}^{\prime}=(2,1,2,3), \mathbf{n}_{5}^{\prime}=(2,2,1,3)$. (See the left hand side of Figure 6 .)
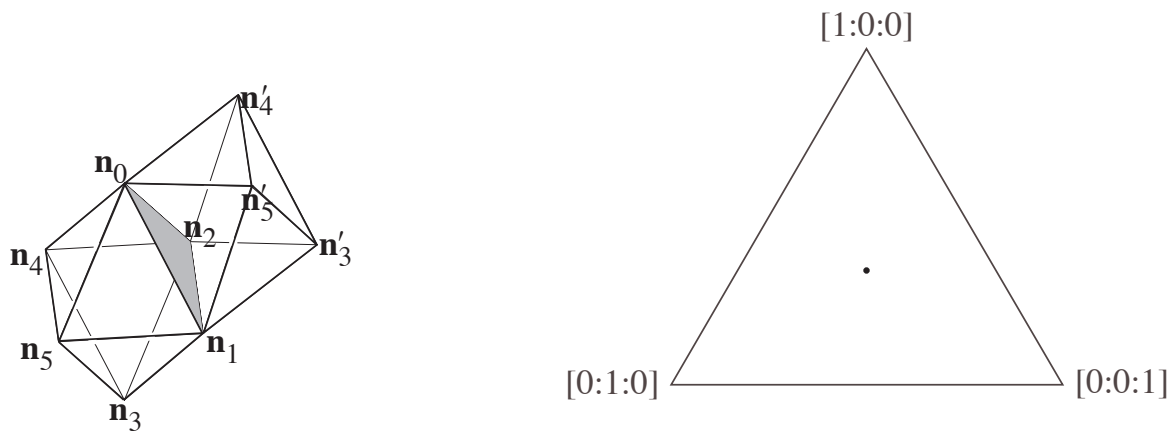

FiguRE 6. Initial cell decomposition of $M$ into ideal octahedra and the corresponding projective parameter cell.

For size vector $v=\left(v_{0}, v_{1}, v_{2}\right)^{t}$ the resulting ideal vertex representatives become $\mathbf{n}_{i} / v_{i}, \mathbf{n}_{i+3} / v_{i}$ and $\mathbf{n}_{i+3}^{\prime} / v_{i}$ for $i=0,1,2$. The hyperplane containing $\mathbf{n}_{0} / v_{0}, \mathbf{n}_{1} / v_{1}$, $\mathbf{n}_{2} / v_{2}$ and $\mathbf{n}_{3} / v_{0}$ is $\left\{\mathbf{x} \mid \mathbf{x} \cdot N_{v}=1\right\}$ where $N_{v}$ is calculated as in the proof of Proposition 6.1 giving

$$
N_{v}=\frac{1}{2}\left(\begin{array}{rrrr}
1 & 0 & 0 & -1 \\
-1 & 2 & 0 & -1 \\
-1 & 0 & 2 & -1 \\
1 & 0 & 0 & 1
\end{array}\right)\left(\begin{array}{l}
v_{0} \\
v_{1} \\
v_{2} \\
v_{0}
\end{array}\right)=\left(\begin{array}{rrr}
0 & 0 & 0 \\
-1 & 1 & 0 \\
-1 & 0 & 1 \\
1 & 0 & 0
\end{array}\right) v
$$

Then $\mathbf{n}_{4} / v_{1}$ and $\mathbf{n}_{5} / v_{2}$ lie in this hyperplane if and only if $\mathbf{n}_{4} \cdot N_{v}-v_{1}=0=$ $(2,-2,0) v$ and $\mathbf{n}_{5} \cdot N_{v}-v_{2}=0=(2,0,-2) v$. Hence $L_{C}=\left(\begin{array}{rrr}2 & -2 & 0 \\ 2 & 0 & -2\end{array}\right)$. By symmetry, $L_{C^{\prime}}$ is the same. Next we compute the tilt of $f_{0}$, the face between $C$ and $C^{\prime}$. This will be positive if $\mathbf{n}_{3}^{\prime} / v_{0}$ lies above the hyperplane defined by $N_{v}$, i.e. 
if $\mathbf{n}_{3}^{\prime} \cdot N_{v}-v_{0}=(-2,2,2) v>0$. Thus $F_{f_{0}}=(-2,2,2)$, and the tilt is positive at $v=(1,1,1)^{t}$. By symmetry, the other faces of the octahedra also have positive tilt. The parameter cell of this decomposition $\left\{v \mid v_{0}=v_{1}=v_{2}\right\}$ is, projectively, a point (as shown in the right half of Figure 6).

Suppose next we increase $v_{0}$ slightly. The faces of $C$ still have positive tilt. We claim that $C$ is subdivided into 4 tetrahedra containing the edge $\mathbf{n}_{0}, \mathbf{n}_{3}$ as shown in the left of Figure 7 below.
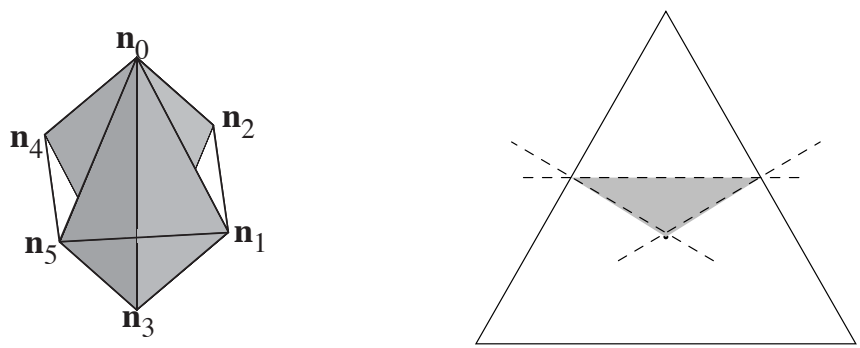

FiguRE 7. Decomposition into ideal tetrahedra and corresponding parameter cell.

Let $f_{1}$ be the triangle with vertices $\left\{\mathbf{n}_{0}, \mathbf{n}_{2}, \mathbf{n}_{3}\right\}$. Then $f_{1}$ has positive tilt if and only if $\mathbf{n}_{4} \cdot N_{v}-v_{1}=(2,-2,0) v=F_{f_{1}} v>0$. Letting $f_{2}$ be the triangle with vertices $\left\{\mathbf{n}_{0}, \mathbf{n}_{1}, \mathbf{n}_{3}\right\}$, we obtain $F_{f_{2}}=(2,0,-2)$. By symmetry, the tilts of the other two faces shown above are the same. Four more triangles, having the same tilts, subdivide $C^{\prime}$. Therefore $M$ is subdivided into 8 simplices in the parameter cell shown in the right of Figure 7 .

Increasing $v_{0}$, eventually $F_{f_{0}} v=0$, so that the faces of $C$ and $C^{\prime}$ vanish from the decomposition. The four simplices around the edge $\mathbf{n}_{1}, \mathbf{n}_{2}$ become an octahedron. Further increasing $v_{0}$ so $F_{f_{0}} v<0$, we claim that this octahedron splits at the square dual to the edge $\mathbf{n}_{1}, \mathbf{n}_{2}$ into two square-based pyramids with vertices $\left\{\mathbf{n}_{0}, \mathbf{n}_{0}^{\prime}, \mathbf{n}_{3}^{\prime}, \mathbf{n}_{3}, \mathbf{n}_{1}\right\}$ and $\left\{\mathbf{n}_{0}, \mathbf{n}_{0}^{\prime}, \mathbf{n}_{3}^{\prime}, \mathbf{n}_{3}, \mathbf{n}_{2}\right\}$ as shown in the left of Figure 8 .
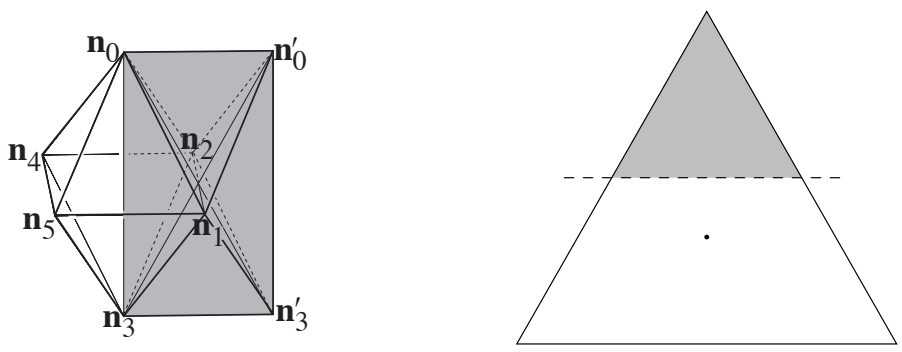

FiguRE 8. Decomposition into square-based pyramids and corresponding parameter cell.

Let $N_{v}^{\prime}$ define the hyperplane containing $\left\{\mathbf{n}_{0} / v_{0}, \mathbf{n}_{1} / v_{1}, \mathbf{n}_{3} / v_{0}, \mathbf{n}_{3}^{\prime} / v_{0}\right\}$. Then

$$
N_{v}^{\prime}=\left(\begin{array}{rrr}
0 & 0 & 0 \\
-1 & 1 & 0 \\
0 & -1 & 0 \\
1 & 0 & 0
\end{array}\right) v
$$

Let $C_{1}$ be the square-based pyramid with vertices $\left\{\mathbf{n}_{0}, \mathbf{n}_{1}, \mathbf{n}_{3}, \mathbf{n}_{3}^{\prime}, \mathbf{n}_{0}^{\prime}\right\}$, where $\mathbf{n}_{0}^{\prime}=$ $(-1,2,2,3)$ is the Minkowski reflection of $\mathbf{n}_{3}^{\prime}$ in the plane containing $\mathbf{n}_{1}, \mathbf{n}_{2}, \mathbf{n}_{4}, \mathbf{n}_{5}$. 
We find that $L_{C_{1}}=(0,0,0)$. Let $f_{3}$ be the triangle with vertices $\left\{\mathbf{n}_{0}, \mathbf{n}_{3}, \mathbf{n}_{3}^{\prime}\right\}$. Then $F_{f_{3}}=(1,-1,-1)$ and $F_{f_{3}} v>0$. Therefore $M$ is divided into 4 square-based pyramids in the parameter cell shown in the right of Figure 8 as mentioned in Remark 6.3.

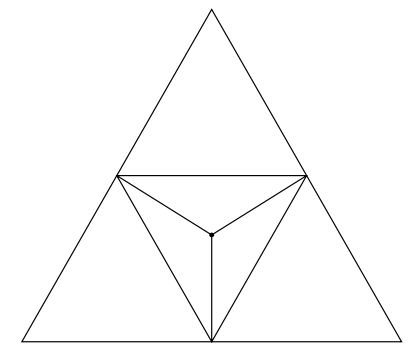

FIGURE 9. All parameter cells for canonical decompositions of the Borromean rings complement

By symmetry, the full set of parameter cells is projectively as shown in Figure 9. On the three line segments containing the centre point, each of the original octahedra is subdivided into two square based pyramids.

\section{Commensurability of Cusps}

The number of canonical cell decompositions that can arise for a multi-cusped manifold can be quite large, placing practical limits on the usefulness of our methods. We show next how it is often possible to greatly reduce the complexity of computing the commensurator of a manifold.

A horospherical cross section of a cusp in an orientable hyperbolic 3-manifold is a Euclidean torus, well defined up to similarity. We can position and scale a fundamental parallelogram in $\mathbb{C}$ such that one vertex lies at the origin and the two adjacent edges end at 1 and a point $z$ in the upper half-plane. Such a $z$ is called a cusp shape parameter. Alternative choices of fundamental parallelogram yield parameters differing by the action of $\mathrm{SL}_{2} \mathbb{Z}$ by Möbius transformations. By choosing a suitable fundamental domain for the action of $\mathrm{SL}_{2} \mathbb{Z}$ on the upper halfplane, we can make a canonical choice of shape parameter for each cusp.

If one cusp covers another, there is an induced covering of Euclidean tori. It follows that their cusp shape parameters are related by the action of an element of $\mathrm{GL}_{2} \mathbb{Q}$. Let us call cusp shapes commensurable if they are so related. As we shall see shortly, it is not hard to determine when two cusp shapes are commensurable.

Suppose we are trying to determine the (discrete) commensurator quotient $Q$ of $M$. If $M$ has cusps of incommensurable shape, these necessarily cover distinct cusps of $Q$. Therefore any assignment of cusp neighbourhoods in $Q$ will yield a Ford spine and tiling whose symmetry group is the whole commensurator (the group of covering transformations of $Q$ ). It follows that we can start by choosing arbitrary horospheres in each of a set of representatives for the commensurability classes of cusps of $M$. Then as we vary our choices of horosphere in the remaining cusps we will be sure to find a tiling whose symmetry group is the commensurator of $M$. The easiest case is when no two cusps of $M$ are commensurable. Then any choice of horospheres at all will do.

The harder case is when $M$ has multiple commensurable cusps. If the symmetry group of $M$ is non-trivial, it may act by non-trivially permuting some of these cusps. Conceptually we should first divide $M$ by its symmetry group and then find the commensurator of this quotient. For practical purposes it is hard to work with 
non-manifold quotients. Instead, whenever two cusps are related by a symmetry of $M$, we choose symmetrically equivalent cusp neighbourhoods. Let $c$ be the number of orbits under the action of $\operatorname{Symm}(M)$ on the cusps. Let $d$ be the number of distinct commensurability classes of cusp shape. Then the parameter space of relative horosphere positions we need to search, in order to find a tiling whose symmetry group equals the commensurator, has dimension $c-d$.

Our algorithm is really only a slight modification of the algorithm of Weeks 33 . for finding the symmetries of a cusped hyperbolic manifold $M=\mathbb{H}^{3} / \Gamma$. In order to find the symmetries we need only consider the canonical cell decomposition arising from a choice of cusp neighbourhoods such that all boundary tori have equal area. Then the symmetries of $M$ are the symmetries of the lifted tiling that normalize $\Gamma$. Equivalently, they are the symmetries of the tiling for which the covering described in Remark 4.2 has degree one.

Returning to the question of when two cusp shapes are commensurable, we note first that cusp shapes of $M$ belong to the invariant trace field of $M$. But if $k$ is any number field, and $\alpha, \alpha^{\prime}$ are irrational elements of $k$, they are related by an element of $\mathrm{GL}_{2} \mathbb{Q}$ if and only if

$$
(c \alpha+d) \alpha^{\prime}=a \alpha+b
$$

is soluble for $a, b, c, d \in \mathbb{Q}$ such that

$$
a d-b c \neq 0 .
$$

We can replace (2) with the condition that $a, b, c, d$ are not all zero, since for $\alpha, \alpha^{\prime} \notin \mathbb{Q},(2)$ follows automatically from (1) and the fact that $\{1, \alpha\}$ are linearly independent over $\mathbb{Q}$. Regarding $k$ as a finite dimensional vector space over $\mathbb{Q}$ we see that (1) has non trivial solutions if and only if $\left\{1, \alpha, \alpha^{\prime}, \alpha \alpha^{\prime}\right\}$ are linearly dependent over $\mathbb{Q}$. In particular, if $[k: \mathbb{Q}]<4$ all irrationals are commensurable in this sense. We thank Ian Agol for pointing out this condition.

7.1. Example: a knot with cusp field not equal to invariant trace field. An interesting example uncovered during this work is the complement of the knot $12 n 706$ shown below. This has one torus cusp with shape parameter $z=6 i$ generating a cusp field $\mathbb{Q}(i)$ which is strictly contained in its invariant trace field $\mathbb{Q}(i, \sqrt{3})$. This answers a question of Neumann-Reid in [27, who asked whether the figure eight knot and the two dodecahedral knots of Aitchison-Rubinstein 3 were the only such examples ${ }^{2}$.

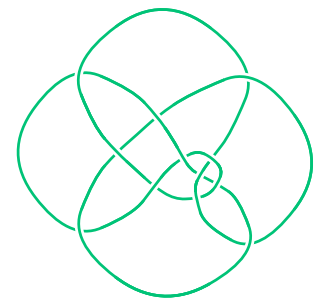

\section{Experimental Results}

We have implemented the algorithms described here and used them to compute the commensurability classes of all 4929 manifolds in the Hildebrand-Weeks census of cusped hyperbolic 3-manifolds [9] and all 7969 complements of the 8614

\footnotetext{
${ }^{2}$ Alan Reid informs us that Nathan Dunfield has found another example: the 15 crossing knot $15 n 132539$.
} 
hyperbolic knots and links up to 12 crossings. After replacing non-orientable manifolds (in the 5-census) by their double covers and removing duplicates we obtained a total of 12783 orientable manifolds. Tables of all the results are available at http://www.ms.unimelb.edu.au/ snap/

In the process it was necessary to identify the arithmetic manifolds and arrange them into their own separate commensurability classes. Criteria for arithmeticity and for commensurability of arithmetic manifolds are given in [10] and [23. In fact, a cusped manifold is arithmetic if and only if its invariant trace field is imaginary quadratic and it has integer traces. Cusped arithmetic manifolds are commensurable if and only if they have the same invariant trace field. They are therefore classified by the discriminant $d$ of the invariant trace field $\mathbb{Q}(\sqrt{d})$.

There were 142 arithmetic manifolds in six commensurability classes. Table 1 lists some of the arithmetic manifolds found with discriminant $-3,-4$ or -7 , and all with discriminant $-8,-11$ or -15 .

\begin{tabular}{ll}
$d$ & manifolds \\
\hline-3 & $\begin{array}{l}\text { m000, m002, m003, m004=4a1, m025, m203=6a5=11n318, s118, } \\
\text { s961, 8a39, 10a280, 10n130, 10n143, 10n155, 11n539, 12a3285, . }\end{array}$ \\
\hline-4 & $\begin{array}{l}\mathrm{m} 001, \mathrm{~m} 124=8 \mathrm{n} 10=10 \mathrm{n} 139, \mathrm{~m} 125, \mathrm{~m} 126, \mathrm{~m} 127, \mathrm{~m} 128, \mathrm{~m} 129, \mathrm{~s} 859, \\
\text { v1858, 8n8=9n34=10n112, 9n36, 10a242, 10n74, 11n545, . }\end{array}$ \\
\hline-7 & $\begin{array}{l}\mathrm{m} 009, \mathrm{~m} 010, \mathrm{~s} 772, \mathrm{~s} 773, \mathrm{~s} 774, \mathrm{~s} 775, \mathrm{~s} 776=6 \mathrm{a} 8=8 \mathrm{n} 7,8 \mathrm{a} 25,8 \mathrm{a} 37, \\
\text { 10n73=12n968, 10n113, 11n498, 12n3068, 12n3078, 12n3093, .. }\end{array}$ \\
\hline-8 & v2787, v2788, v2789, 9a73, 9a74, 12a3292, 12a3296, 12n2625= \\
\hline-11 & $12 \mathrm{n} 2630,12 \mathrm{n} 2972,12 \mathrm{n} 3088,12 \mathrm{n} 3098=12 \mathrm{n} 3099$. \\
\hline-15 & $12 \mathrm{a} 3169,12 \mathrm{a} 3273,12 \mathrm{a} 3284,12 \mathrm{a} 3300,12 \mathrm{a} 3307,12 \mathrm{a} 3308$.
\end{tabular}

TABLE 1. Selected arithmetic manifolds.

The naming of manifolds in the tables is as follows. Manifolds whose names begin with $\mathrm{m}$, s or $\mathrm{v}$ belong to the 5,6 or 7 tetrahedra census of cusped manifolds respectively. The rest are knot and link complements in the form < number of crossings $><$ alternating or non-alternating $><$ index in table $>$. The link tables were provided by Morwen Thistlethwaite and are included with current versions of Snap [16] and Tube [17.

The remaining 12641 manifolds were non-arithmetic, falling into 11709 commensurability classes. A few of them are shown in Table 2 .

Column headings are as follows: s.g. is the order of the symmetry group; c.d. is the degree of the manifold over its commensurator quotient; c.vol is the volume of the commensurator quotient, optionally followed by c.cl, commensurability class numbered from 0 , when incommensurable manifolds are listed with the same commensurator volume. Thus manifolds are commensurable if and only if they have the same entry in this column. The invariant trace field is described by its degree, discriminant and a number specifying which root of the minimum polynomial generates it (with sign corresponding to choice of complex conjugate); nc,cnc gives the number of cusps in the manifold and the number of cusps in the commensurator quotient; cusp density is computed using equal area cusps in the commensurator quotient.

The first group of manifolds have the smallest commensurator volume found (among non-arithmetic manifolds) and are the link complements which appeared in Section 2.1. They all have 'hidden symmetries,' i.e. commensurabilities not arising from the symmetry group of the manifold. The total number of non-arithmetic 


\begin{tabular}{lrrllll} 
manifold & s.g. & c.d. & c.vol,c.cl & field & nc,cnc & cusp density \\
\hline 10a297 & 20 & 40 & 0.365076519 & $4,1025(2)$ & 5,1 & 0.642619992 \\
10a291 & 4 & 40 & 0.365076519 & $4,1025(2)$ & 4,1 & 0.642619992 \\
10a277 & 4 & 40 & 0.365076519 & $4,1025(2)$ & 3,1 & 0.642619992 \\
12n2492 & 4 & 40 & 0.365076519 & $4,1025(2)$ & 3,1 & 0.642619992 \\
12n2899 & 4 & 40 & 0.365076519 & $4,1025(2)$ & 4,1 & 0.642619992 \\
\hline 12n1189 & 2 & 2 & $7.175483613,0$ & $7,-76154488(-2)$ & 2,2 & 0.589830477 \\
12n1190 & 2 & 2 & $7.175483613,1$ & $7,-76154488(-2)$ & 2,2 & 0.589830477 \\
12n1481 & 2 & 2 & $7.175483613,2$ & $7,-76154488(-2)$ & 2,2 & 0.589830477 \\
12n2348 & 2 & 2 & $7.175483613,3$ & $7,-76154488(-2)$ & 3,3 & 0.644747497 \\
12n2580 & 2 & 2 & $7.175483613,4$ & $7,-76154488(-2)$ & 3,3 & 0.631898787 \\
\hline m045 & 4 & 4 & 0.818967911 & $3,-107(-2)$ & 1,1 & 0.608307263 \\
m046 & 4 & 4 & 0.818967911 & $3,-107(-2)$ & 1,1 & 0.608307263 \\
v3379 & 8 & 8 & 0.818967911 & $3,-107(-2)$ & 2,1 & 0.608307263 \\
v3383 & 4 & 8 & 0.818967911 & $3,-107(-2)$ & 3,1 & 0.608307263 \\
v3384 & 8 & 8 & 0.818967911 & $3,-107(-2)$ & 2,1 & 0.608307263 \\
12a1743 & 8 & 16 & 0.818967911 & $3,-107(-2)$ & 2,1 & 0.608307263 \\
v3376 & 4 & 4 & $1.637935822,0$ & $3,-107(-2)$ & 2,1 & 0.690189995 \\
v3377 & 4 & 4 & $1.637935822,0$ & $3,-107(2)$ & 1,1 & 0.690189995 \\
v3378 & 4 & 4 & $1.637935822,0$ & $3,-107(2)$ & 1,1 & 0.690189995 \\
12a2937 & 8 & 8 & $1.637935822,1$ & $6,-1225043(1)$ & 3,2 & 0.608307263 \\
\hline 9a94 & 4 & 24 & 0.575553268 & $4,144(1)$ & 3,2 & 0.844133714 \\
& & TABLE 2. Selected non-arithmetic manifolds. &
\end{tabular}

manifolds having hidden symmetries was 148 . The next group of manifolds shows that incommensurable manifolds are not always distinguished by cusp density. The third group of manifolds includes manifolds whose classes are distinguished by invariant trace field but not by cusp density, and manifolds with the same commensurator quotient volume but different invariant trace fields. One should not get the impression that cusp density is a poor invariant: in fact, among the 11278 cusp densities found, only 417 grouped together incommensurable manifolds. The final line gives data for the non-arithmetic manifold with highest cusp density found. (The maximum possible cusp density is $0.853276 \ldots$, which occurs for the figure eight knot complement.)

More details of the fields occurring above are listed in the Table 3

\begin{tabular}{crcl} 
degree & discriminant & signature & minimum polynomial \\
\hline 4 & 1025 & 0,2 & $x^{4}-x^{3}+3 x^{2}-2 x+4$ \\
7 & -76154488 & 1,3 & $x^{7}-2 x^{6}+3 x^{5}-5 x^{4}+x^{3}-8 x^{2}-2 x-4$ \\
3 & -107 & 1,1 & $x^{3}-x^{2}+3 x-2$ \\
6 & -1225043 & 0,3 & $x^{6}-2 x^{5}-2 x^{3}+30 x^{2}-52 x+29$ \\
4 & 144 & 0,2 & $x^{4}-x^{2}+1$
\end{tabular}

TABLE 3. Fields in previous table.

\section{Appendix}

The indexing system used for knots and links, here and in snap, may still be subject to change. This is due to the difficulty of determining whether two nonhyperbolic links are equivalent and the consequent possibility that duplicates will later be discovered and removed from the tables. 
For this reason we provide Table 4, giving the Dowker-Thistlethwaite codes of all the knots and links that we refer to. Since, for links, this code is not well known, we describe here how this works.

Firstly there is the trivial matter of passing between the alphabetic codes used by snap, and their numerical forms. The Dowker code for a link with $n$ crossings and $k$ components is a permutation of the even integers $2, \ldots, 2 n$, with possible sign changes, bracketed into $k$ subsequences: e.g. $(6,-8)(-10,14,-12,-16,-2,4)$. To express this alphabetically we encode $n$ and $k$ as the first two letters using $a=$ $1, b=2$, etc. Then follow $k$ letters giving the lengths of the bracketed subsequences. Finally there are $n$ letters giving the sequence of even integers using $a=2, b=4$, etc. and $A=-2, B=-4$, etc. The alphabetic code for the above example is thus hbbfcDEgFHAb.

To go from a link diagram to its Dowker code, proceed as follows. Traverse each component, numbering the crossings, starting with 1 on an overcrossing. When the first component is done, continue with consecutive numbers on the next component. Each crossing will receive two numbers. We can number the crossings in such a way that every crossing gets one even and one odd number. (This follows easily from the fact that we can two-colour the plane containing a link diagram.) Negate any even number which labels an over-crossing (for an alternating link there will not be any). For each odd number $1,3, \ldots, 2 n-1$ write down the corresponding even number: this gives a sequence of $n$ even numbers. A component with $2 j$ crossings will have $j$ odd numbers on it, so there will be $j$ corresponding numbers for it in the code; bracket together the numbers for each component. See for example Figure 10 .

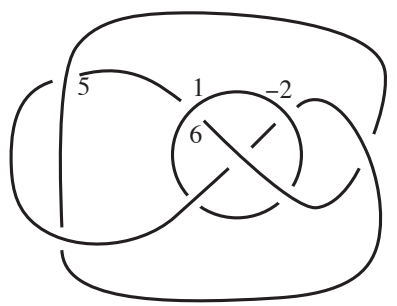

Figure 10. Complete the numbering indicated on the above two-component link to obtain the Dowker-Thistlethwaite code $(6,-8)(-10,14,-12,-16,-2,4)$.

The reverse procedure, going from a code to a link diagram is a little bit more tricky, but essentially the same as the procedure for knots, described in [2]. We draw the first component as a knot, with extra as-yet-unconnected crossings on it. When adding further components we will encounter crossings with other link components. 


\begin{tabular}{|c|c|c|c|}
\hline name & Dowker code & name & Dowker code \\
\hline $4 \mathrm{a} 1$ & dadbcda & $12 \mathrm{a} 2937$ & lcbeechjklaiefgbd \\
\hline $6 a 5$ & fbccdefacb & $12 \mathrm{a} 2961$ & lcbfdcfahibekdlgj \\
\hline $6 a 8$ & fcbbbceafbd & $12 \mathrm{a} 3039$ & lcbhbcfaikbjelgdh \\
\hline $8 \mathrm{a} 25$ & hbbfcfegahdb & $12 \mathrm{a} 3169$ & lccfcdfhcjakblieg \\
\hline $8 \mathrm{a} 37$ & hcbcccfghadeb & $12 \mathrm{a} 3230$ & ldbccdcfagiebkdlhj \\
\hline $8 a 39$ & hdbbbbceagbhdf & $12 \mathrm{a} 3273$ & ldbddbceagbidkflhj \\
\hline $8 \mathrm{n} 7$ & hcbcccDFgHEaB & $12 \mathrm{a} 3284$ & ldbfbbceaibkjldgfh \\
\hline $8 \mathrm{n} 8$ & hcbcccdFgHEab & $12 \mathrm{a} 3285$ & ldbfbbcfaikbjlehdg \\
\hline $8 \mathrm{n} 10$ & hdbbbbcEaGBHDF & $12 \mathrm{a} 3292$ & ldccccdegjhkflacib \\
\hline $9 \mathrm{a} 73$ & ibcfdfhagbice & $12 \mathrm{a} 3295$ & ldccccdgjakhbflice \\
\hline $9 \mathrm{a} 74$ & ibcfdfhaibecg & $12 \mathrm{a} 3296$ & ldccccdgjhkbelcfia \\
\hline $9 \mathrm{a} 94$ & icbebcdhfiabeg & $12 \mathrm{a} 3300$ & lebbbdbceagbidkflhj \\
\hline 9 n34 & icbdcceaGbHDIF & $12 \mathrm{a} 3307$ & lebccbbcfaikbljdheg \\
\hline 9 n36 & icbebcdHfiabeG & $12 \mathrm{a} 3308$ & lfbbbbbbceagbidkflhj \\
\hline $10 \mathrm{a} 242$ & jbeefghjiaecbd & $12 \mathrm{n} 706$ & lalceFhGjIKlBaD \\
\hline $10 \mathrm{a} 277$ & jcbfbceagbidjfh & $12 \mathrm{n} 968$ & lbbjcDFhIJLAbKEG \\
\hline $10 \mathrm{a} 280$ & jcccddeghjibcfa & $12 \mathrm{n} 1189$ & lbbjceaHbKiDjgLF \\
\hline $10 \mathrm{a} 291$ & jdbbdbceagbidjfh & $12 \mathrm{n} 1190$ & lbbjceaHbkIDJGlf \\
\hline $10 \mathrm{a} 297$ & jebbbbbceagbidjfh & $12 \mathrm{n} 1481$ & lbbjchaEGJDbkFli \\
\hline $10 \mathrm{n} 73$ & jbbhcEFihGJAdb & $12 \mathrm{n} 1848$ & lbcidfIaglceKBHJ \\
\hline $10 \mathrm{n} 74$ & jbbhcfaHGIbDJE & $12 \mathrm{n} 2348$ & lcbbhcEaHBKiDjgLF \\
\hline 10n112 & jcbcecdFgIHabJE & $12 \mathrm{n} 2492$ & lcbdfceaGbiDJKfLH \\
\hline 10n113 & jcbcecFaHJIBDGE & $12 \mathrm{n} 2580$ & lcbeecHaEGJDBkFli \\
\hline 10n130 & jcbfbceaGbIDJFH & $12 \mathrm{n} 2625$ & lcbfdcfaIJbKLEDHG \\
\hline 10n139 & jdbbcccEaHBijDgf & $12 \mathrm{n} 2630$ & lcbfdcfaIJbKLHDEG \\
\hline 10n143 & jdbbdbcEaGBiDjfh & $12 \mathrm{n} 2899$ & ldbbbfcEaGBIDjkFlh \\
\hline 10n155 & jebbbbbcEaGBIDjFh & $12 \mathrm{n} 2972$ & ldbccdcfaIJbKLEDHG \\
\hline $11 n 318$ & kbchdEfcHiJKaBG & 12 n3068 & ldbddbceaGbIDKFLHJ \\
\hline $11 n 498$ & kcbcfcfaHJbIKEGD & 12 n3078 & ldbfbbceaIbKJLDGFH \\
\hline $11 n 539$ & kcbedceaHbIJDGKF & 12 n3088 & ldccccdeGacJBklFih \\
\hline $11 n 545$ & kcbfccdIfJabKGEH & 12 n3093 & ldccccdEGHiJKlAFBc \\
\hline $12 \mathrm{a} 1743$ & lbbjchfjialkedbg & 12 n3098 & ldccccdEGhIJKLaFBC \\
\hline $12 \mathrm{a} 2126$ & lbcidgjclhafkbie & 12n3099 & ldccccdEGhIJKlAFBc \\
\hline
\end{tabular}

TABLE 4. Dowker-Thistlethwaite codes of all knots and links mentioned in this paper.

\section{REFERENCES}

[1] C. Adams, Noncompact hyperbolic 3-orbifolds of small volume, Topology '90 (Columbus, OH, 1990), 1-15, Ohio State Univ. Math. Res. Inst. Publ., 1, de Gruyter, Berlin, 1992.

[2] C. Adams, The Knot Book, W. H. Freeman and Company, New York, 1994.

[3] I. R. Aitchison and J. H. Rubinstein, Combinatorial Cubings, Cusps and the Dodecahedral Knots, Topology '90 (Columbus, OH, 1990), 273-310, Ohio State Univ. Math. Res. Inst. Publ., 1, de Gruyter, Berlin, 1992.

[4] H. Akiyoshi, Finiteness of polyhedral decompositions of cusped hyperbolic manifolds obtained by the Epstein-Penner's method. Proc. Amer. Math. Soc. 129 (2001), no. 8, 24312439 .

[5] H. Akiyoshi, M. Sakuma, M. Wada and Y. Yamashita, Punctured Torus Groups and 2Bridge Knot Groups (I), Lecture Notes in Mathematics, Vol. 1909, Springer, 2007.

[6] A. Borel, Commensurability classes and volumes of hyperbolic 3-manifolds. Ann. Scuola Norm. Sup. Pisa Cl. Sci. (4) 8 (1981), no. 1, 1-33.

[7] B. H. Bowditch, C. Maclachlan and A. W. Reid, Arithmetic hyperbolic surface bundles. Math. Ann. 302 (1995), no. 1, 31-60. 
[8] J. O. Button, Fibred and virtually fibred hyperbolic 3-manifolds in the censuses. Experiment. Math. 14 (2005), no. 2, 231-255.

[9] P. J. Callahan, M. V. Hildebrand and J. R. Weeks, A census of cusped hyperbolic 3manifolds. (With microfiche supplement.) Math. Comp. 68 (1999), no. 225, 321-332.

[10] D. Coulson, O. Goodman, C. Hodgson and W. Neumann, Computing arithmetic invariants of 3-manifolds, Experimental Mathematics 9 (2000), 127-152.

[11] D. B. A. Epstein and R. C. Penner, Euclidean decompositions of noncompact hyperbolic manifolds. J. Differential Geom. 27 (1988), no. 1, 67-80.

[12] W. Floyd and A. Hatcher, Incompressible surfaces in punctured-torus bundles. Topology and its Applications 13 (1982), 263-282.

[13] F. González-Acuña and W. C. Whitten, Imbeddings of three-manifold groups. Mem. Amer. Math. Soc. 99 (1992), no. 474, viii+55 pp.

[14] F. Guéritaud (with an appendix by D. Futer), On canonical triangulations of once-punctured torus bundles and two-bridge link complements, Geometry and Topology 10 (2006), pp. 1239-1284.

[15] F. Guéritaud, Géométrie hyperbolique effective et triangulations idéals canoniques en dimension 3, Thèse de doctorat, Univ. Paris-sud, Orsay, 2006

[16] O. Goodman, Snap, the computer program, http://www.ms.unimelb.edu.au/ ${ }^{\sim}$ snap/ and http://sourceforge.net/projects/snap-pari.

[17] O. Goodman, Tube: a computer program for studying geodesics in hyperbolic 3-manifolds, available from http://www.ms.unimelb.edu.au/ ${ }^{\sim} \operatorname{snap} /$.

[18] D. Heard, Orb: a computer program for studying hyperbolic 3-orbifolds, available from http://www.ms.unimelb.edu.au/ ${ }^{\sim}$ snap/orb.html.

[19] M. Hildebrand and J. Weeks, A computer generated census of cusped hyperbolic 3-manifolds. Computers and mathematics (Cambridge, MA, 1989), 53-59, Springer, New York, 1989.

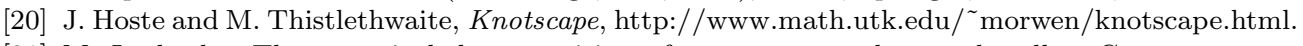

[21] M. Lackenby, The canonical decomposition of once-punctured torus bundles, Comment. Math. Helv. 78 (2003) 363-384.

[22] A. M. Macbeath, Commensurability of co-compact three-dimensional hyperbolic groups. Duke Math. J. 50 (1983), no. 4, 1245-1253. Erratum: Duke Math. J. 56 (1988), no. 1, 219.

[23] C. Maclachlan and A. Reid, The arithmetic of hyperbolic 3-manifolds, Springer-Verlag, New York, 2003.

[24] G. A. Margulis, Discrete subgroups of semisimple Lie groups, Ergebnisse der Mathematik und ihrer Grenzgebiete 17, Springer-Verlag, Berlin, 1991.

[25] T. H. Marshall and G. J. Martin, Minimal Co-Volume Hyperbolic Lattices II, in preparation.

[26] R. Meyerhoff, The cusped hyperbolic 3-orbifold of minimal volume, Bull. Amer. Math. Soc. 13 (1985), 154-156.

[27] W. D. Neumann and A. W. Reid, Arithmetic of Hyperbolic Manifolds, Topology '90 (Columbus, OH, 1990), 273-310, Ohio State Univ. Math. Res. Inst. Publ., 1, de Gruyter, Berlin, 1992.

[28] W. D. Neumann and A. W. Reid, Notes on Adams' small volume orbifolds, Topology '90 (Columbus, OH, 1990), 311-314, Ohio State Univ. Math. Res. Inst. Publ., 1, de Gruyter, Berlin, 1992

[29] A. W. Reid, A note on trace-fields of Kleinian groups. Bull. London Math. Soc. 22 (1990), no. $4,349-352$.

[30] M. Sakuma and J. Weeks, Examples of canonical decompositions of hyperbolic link complements. Japan. J. Math. (N.S.) 21 (1995), no. 2, 393-439.

[31] M. Sakuma and J. Weeks, The generalized tilt formula. Geom. Dedicata 55 (1995), no. 2, $115-123$.

[32] W. P. Thurston, The geometry and topology of three-manifolds, Princeton University Math. Dept. (1978). Also available at http://msri.org/publications/books/gt3m.

[33] J. R. Weeks, Convex hulls and isometries of cusped hyperbolic 3-manifolds. Topology Appl. 52 (1993), no. 2, 127-149.

[34] J. Weeks, SnapPea, the computer program, available from http://geometrygames.org/SnapPea/index.html.

[35] R. Zimmer, Ergodic theory and semi-simple Lie groups, Birkhauser, Boston, 1984.

Department of Mathematics and Statistics, University of Melbourne, Parkville, VicTORIA 3010, Australia

E-mail address: oag@optusnet.com.au, damian.heard@gmail.com, cdh@ms.unimelb.edu.au 